\title{
Trafficking receptor signatures define blood plasmablasts responding to tissue-specific immune challenge
}

\author{
Yekyung Seong, ${ }^{1,2}$ Nicole H. Lazarus, ${ }^{1,3}$ Lusijah Sutherland, ${ }^{1,3}$ Aida Habtezion, ${ }^{4}$ Tzvia Abramson, ${ }^{5}$ \\ Xiao-Song He, ${ }^{3,6}$ Harry B. Greenberg, ${ }^{3,6,7}$ and Eugene C. Butcher ${ }^{1,3}$ \\ 'Laboratory of Immunology and Vascular Biology, Department of Pathology, Stanford University School of Medicine, \\ Stanford, California, USA and the Veterans Affairs Palo Alto Health Care System, Palo Alto, California, USA. ${ }^{2}$ Program of \\ Immunology, Stanford University School of Medicine, Stanford, California, USA. ${ }^{3}$ The Center for Molecular Biology and \\ Medicine, Veterans Affairs Palo Alto Health Care System, Palo Alto, California, USA. ${ }^{4}$ Division of Gastroenterology and \\ Hepatology, Stanford University School of Medicine, Stanford, California, USA. ${ }^{5}$ San Jose State University, Department of \\ Biology, San Jose, California, USA. ${ }^{6}$ Department of Medicine, Stanford University School of Medicine, Stanford, California, \\ USA. 'Department of Immunology and Microbiology, Stanford University School of Medicine, Stanford, California, USA.
}

Antibody-secreting cells are generated in regional lymphoid tissues and traffic as plasmablasts (PBs) via lymph and blood to target sites for local immunity. We used multiparameter flow cytometry to define PB trafficking programs (TPs, combinations of adhesion molecules and chemoattractant receptors) and their imprinting in patients in response to localized infection or immune insults. TPs enriched after infection or autoimmune inflammation of mucosae correlate with sites of immune response or symptoms, with different TPs imprinted during small intestinal, colon, throat, and upper respiratory immune challenge. PBs induced after intramuscular or intradermal influenza vaccination, including flu-specific antibody-secreting cells, display TPs characterized by the lack of mucosal homing receptors. PBs of healthy donors display diverse mucosa-associated TPs, consistent with homeostatic immune activity. Identification of TP signatures of PBs may facilitate noninvasive monitoring of organ-specific immune responses.

Authorship note: Y. Seong, N.H. Lazarus, and L. Sutherland are co-first authors, having contributed equally to this work.

Conflict of interest: The authors have declared that no conflict of interest exists.

Submitted: August 23, 2016

Accepted: February 9, 2017

Published: March 23, 2017

\section{Reference information:}

JCI Insight. 2017;2(6):e90233. https:// doi.org/10.1172/ji.insight.90233.

\section{Introduction}

Plasmablasts (PBs) are generated in lymphoid tissues associated with sites of antigen challenge, and circulate via the lymphatic and blood circulation to home to target tissues where they undergo further maturation into antibody-secreting plasma cells (ASCs). PBs are present in low numbers in the peripheral blood of healthy individuals. After acute infection or in settings of active immune responses, however, the frequency of blood PBs increases dramatically, peaking 7 to 9 days after immunization or the onset of illness (1-3) and reflects the dissemination of recently antigen-activated B cells (4, 5; reviewed in refs. 6, 7). Circulating PBs then home to target tissues where they further differentiate into mature ASCs.

Early studies of IgA ASC homing from the blood to the gastrointestinal tract showed that recruitment is tissue selective but independent of antigen specificity (4), and it is now widely accepted that homing is mediated by specialized combinations of adhesion and chemoattractant receptors (herein referred to as trafficking programs or TPs) that mediate interactions of circulating PBs with vascular endothelium in target tissues. Studies in the mouse have implicated $\alpha 4 \beta 7$ and its vascular ligand mucosal vascular addressin cell adhesion molecule 1 (MAdCAM1) in IgA ASC localization and function in intestinal immunity (8); CCR10 and its mucosal chemokine ligand CCL28 in IgA PB homing to the lactating mammary gland (9) and to the intestines $(10,11)$, especially to the colon $(12,13)$; and CCR9 and its ligand CCL25 in small intestinal homing $(10,14)$. In humans, studies of blood-borne PBs after intestinal rotavirus (1) or Shigella (3) infection or following experimental intestinal immunization $(15,16)$ have shown high-level expression of intestinal trafficking receptors that target antigen-specific IgA ASCs to the intestines, where they locally produce secretory $\operatorname{IgA}$ at the sites of infection or antigen administration. In contrast, systemic immunization protocols induce ASCs with higher expression of CD62L (L-selectin) $(15,16)$. These studies showed that different homing receptors are imprinted on PBs as a function of immune challenge in the small intestines versus subcutaneous tissues, 

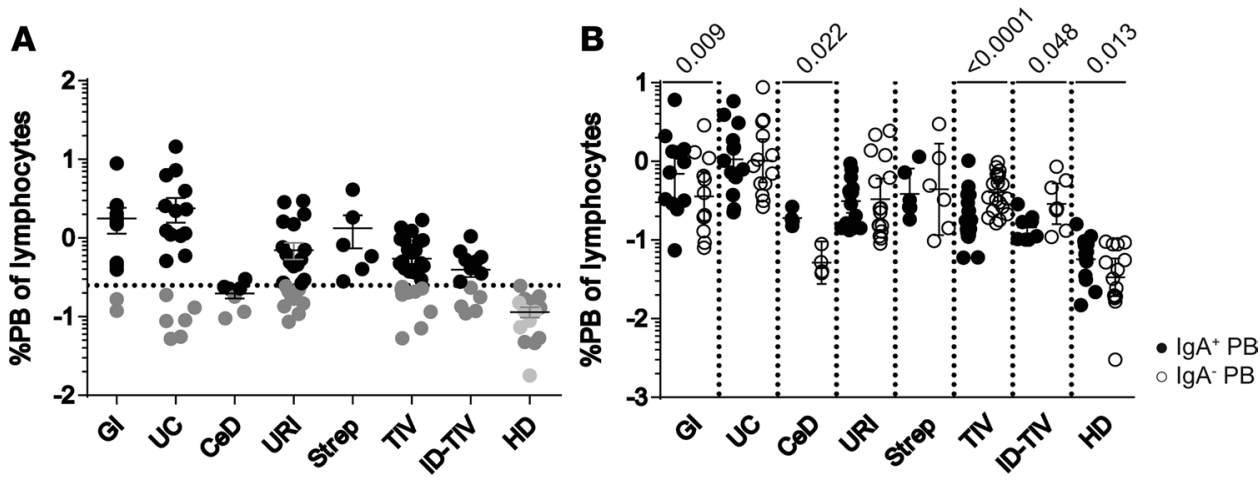

Figure 1. Plasmablast frequency in donor blood. (A) Percentage of plasmablasts (PBs) (log scale) among total lymphocytes. Black symbols represent patients with PB frequencies $\geq 0.25 \%$, dark gray symbols are samples with PBs below $0.25 \%$. (B) IgA $\mathrm{A}^{+}$and IgA- (predominantly IgG) PB percentages. The statistical significance of $\lg \mathrm{A}^{+}$versus IgA- frequencies within a patient class is indicated (paired ratio, 2-tailed $t$ test). For each patient class, the total number of samples (and those with PB frequencies $\geq 0.25 \%$ in parentheses) are as follows: gastrointestinal infection (GI), 13 (8); ulcerative colitis (UC), 19 (5); celiac disease (CeD), 7 (2); upper respiratory infection (URI), 25 (13); streptococcal infection (Strep), 6 (4); intramuscular trivalent influenza vaccination (TIV), 28 (7); intradermal TIV (ID-TIV), 13 (5); healthy donor (HD), 14 (0). but were limited in the range of receptors and clinical settings examined. Importantly, they raised the possibility that specialized TPs might be programmed by the microenvironments in other tissues as well. Elucidation of such TPs might allow identification of circulating PBs participating in immune responses in specific organs and tissues of the body, providing a window on localized immunity.

In this study, we have taken advantage of the surge of bloodborne PBs that appear 7 to 9 days after symptom onset to elucidate TPs of $\mathrm{PBs}$ in volunteers following infectious or inflammatory illnesses localized to mucosal sites or defined systemic immune challenge. Our results reveal a remarkable diversity of TPs

for blood-borne PBs, and define signature TPs that are reproducibly imprinted on PBs during immune responses in the small versus large intestines, the throat, the upper respiratory tract, or in response to intradermal or intramuscular flu vaccination. The identification of imprinted TP signatures may permit noninvasive blood monitoring of antibody responses in various mucosal tissues.

\section{Results}

Elevation of $P B$ numbers in patients. We stained human peripheral blood mononuclear cells (PBMCs) with a cocktail of antibodies to define circulating PBs, and characterized the expression of a set of chemoattractant receptors and adhesion molecules (i.e., TPs). For patients after influenza vaccination, blood was taken 7 days after immunization (17). Patients with ulcerative colitis or celiac disease donated blood samples during periods of active symptoms as assessed by clinical scores (see Methods). Subjects with acute infectious symptoms in intestines or symptoms of food poisoning donated blood samples 7 to 9 days after the symptom onset. These donors provided information about the symptoms of their illness through interview and a questionnaire, including subjective evaluation of the presence, duration, and severity of upper respiratory symptoms (cough, nasal discharge, sore throat), gastrointestinal symptoms (nausea, vomiting, cramping and/or diarrhea/dehydration), and systemic symptoms of fever and myalgia (Supplemental Table 1; supplemental material available online with this article; https://doi.org/10.1172/jci.insight.90233DS1). Patients with similar symptoms or with known diagnoses were grouped into patient classes for analysis; these included patients with gastrointestinal symptoms of unknown etiology (GI), ulcerative colitis (UC), celiac disease $(\mathrm{CeD})$, upper respiratory infectious symptoms (URI), patients with culture-proven streptococcal infection or symptoms limited to sore throat and fever (Strep), and patients after intramuscular trivalent influenza vaccination (TIV) or intradermal TIV (ID-TIV). Healthy donors (HDs), with no history of recent illness, were evaluated for comparison.

We initially assessed the frequency of PBs among PBMCs. PBs were identified by fluorescence gating as $\mathrm{CD} 38^{\text {hi }}$ among $\mathrm{CD}^{+}$lineage marker-negative lymphocytes; the gating strategy is illustrated in Supplemental Figure 1. Total PB frequencies were elevated in many patients compared with healthy controls, but the frequency of PBs in each clinical setting was variable (Figure 1A). In the blood of HDs, evaluated PBs did not exceed $0.25 \%$ of total lymphocytes. This percentage was selected as a cutoff for our analyses of $\mathrm{TP}$ correlation with patient disease and symptoms.

Surface $\operatorname{IgA}^{+}$PBs were gated separately from $\operatorname{IgA}^{-}$PBs. In most settings of infection or influenza vaccination, $\operatorname{IgA}^{-}$PBs are predominantly IgG expressing, although they may comprise IgM ASCs in primary immune responses (2). $\operatorname{IgA}^{+}$and $\operatorname{IgA}^{-}$PBs were generally increased in parallel in patients, although $\mathrm{IgA}^{+} \mathrm{PBs}$ were more frequent in patients with $\mathrm{GI}$ symptoms, $\mathrm{CeD}$, or $\mathrm{HDs}$, whereas $\mathrm{IgA}^{-} \mathrm{PBs}$ were enriched following influenza vaccination (Figure 1B). 
A

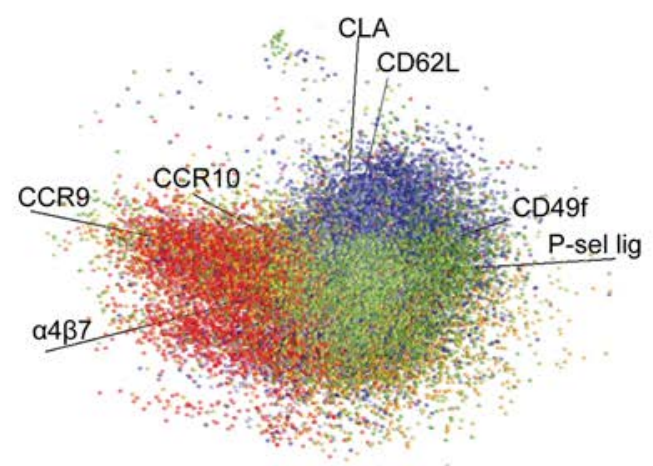

- GI $\odot \mathrm{UC} \odot \mathrm{CeD} \odot \mathrm{URI} \odot \mathrm{Strep} \odot \mathrm{TIV} \odot \mathrm{ID}-\mathrm{TIV}$
B

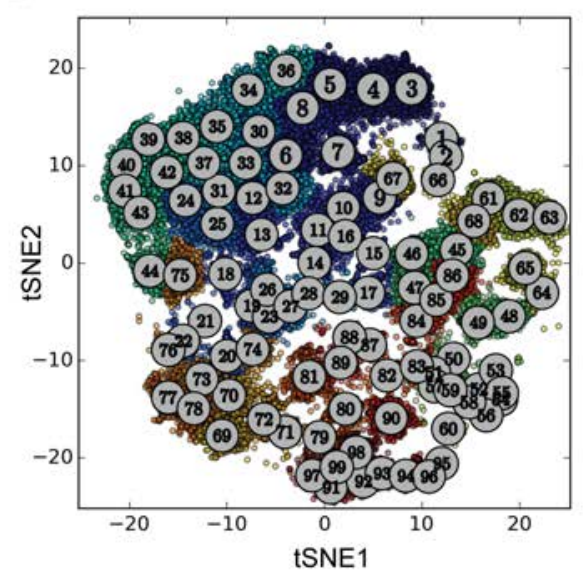

C
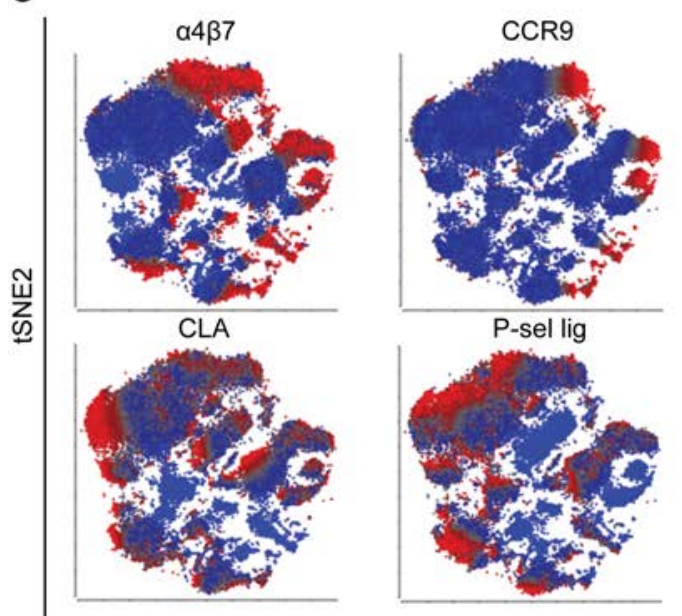
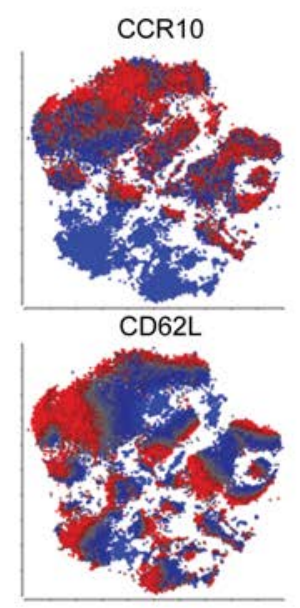

tSNE1

D

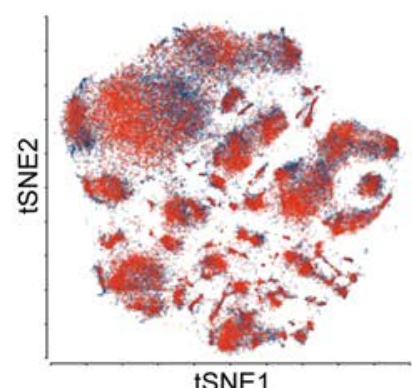

E

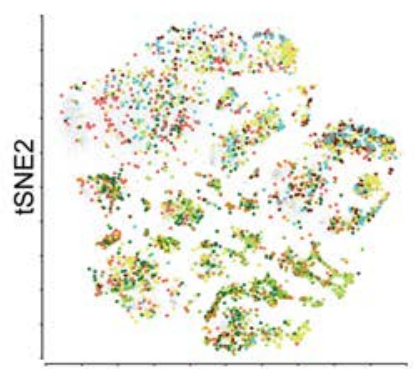

tSNE1

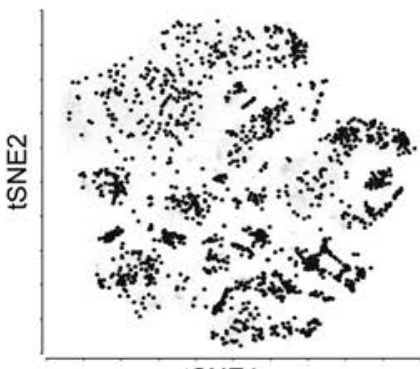

tSNE1

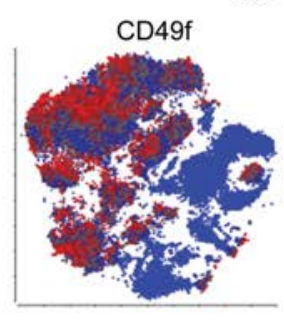

Patterns of trafficking receptor expression define discrete subsets of PBs. The trafficking of blood leukocytes is determined by the specific combinations of adhesion molecules and chemoattractant receptors expressed by individual cells. We used multiparameter flow cytometry to study the patterns of $\mathrm{PB}$ expression of 7 receptors with well-characterized involvement in tissue- or inflammation site-selective lymphocyte recruitment from the blood, or association with selectively

recirculating lymphocyte populations. These included the trafficking receptors integrin $\alpha 4 \beta 7$, receptor for the intestinal vascular addressin MAdCAM1; CCR10, chemoattractant receptor for mucosaassociated epithelial chemokine CCL28; CCR9, receptor for the small intestinal chemokine CCL25; cutaneous lymphocyte associated antigen (CLA), ligand for the vascular adhesion receptor CD62E (E-selectin) expressed in cutaneous sites; and CD62L, the lymphocyte selectin homing receptor for lymph nodes and sites of chronic inflammation. We used a recombinant human P-selectin (CD62P)-Fc chimeric protein to stain for functional binding sites for vascular P-selectin, implicated in leukocyte homing to sites of inflammation. In addition, we stained for CD49f (integrin $\alpha 6$ ), whose function in trafficking is unclear but whose expression distinguishes memory $\mathrm{T}$ cells recirculating through the skin and lung versus gastrointestinal tissues (18). 
We first asked if PBs could be segregated into discrete classes or clusters based on their trafficking receptor profiles. For this analysis, fluorescence intensities of expressed trafficking receptors were exported for 500 individually gated $\mathrm{IgA}^{+} \mathrm{PBs}$ and $500 \mathrm{IgA}^{-} \mathrm{PBs}$ from each patient (fewer for some healthy donors, in whom PB numbers were limiting). Principal component analysis (PCA) of cells based on their trafficking receptor profiles revealed that each of the receptors contributed significantly to the variance in $\mathrm{PB}$ phenotypes. PBs from patients with URI, GI symptoms, and from influenza vaccinees were differently distributed. High CD62L and CLA expression was associated with PBs from patients with URI, and CCR9, CCR10, and $\alpha 4 \beta 7$ with patients with GI symptoms or disease (Figure 2A). However PCA, used singularly, failed to define discrete disease- or symptom-associated trafficking receptor profiles (combinations of receptors).

To define clusters of PBs based on trafficking receptor profiles, we used the Barnes-Hut variant of the t-distributed stochastic neighbor-embedding (tSNE) algorithm. As implemented through automatic classification of cellular expression by nonlinear stochastic embedding (ACCENSE) (19), tSNE supports visualization and statistical evaluation of clustering (20). The algorithm identified well-delineated groups of PBs, indicating that PB TPs are nonrandom (Figure 2, B and C). We used a cluster number of $\sim 100$ to ensure that the cells within each cluster were relatively homogeneous in trafficking receptor profile, while retaining sufficient cells in most clusters to power statistical analysis of cluster association with patient classification. The tSNE-generated clusters are illustrated in Figure 2B. Even the most similar clusters differed 4- to 5-fold in mean fluorescence intensity for at least 1 trafficking receptor and/or 2- to 3-fold for several, differences that are likely to be functionally significant. Although not discussed further, roughly comparable clusters (and downstream results) were obtained using a self-organizing map algorithm.

The results revealed a surprising diversity of expression profiles on circulating PBs. Figure 2C shows the expression of each trafficking receptor as visualized in the tSNE map. Each of the 99 clusters comprised cells from many different patients including HDs (Figure 2, D and E), and most donors' PBs comprised cells from many if not all clusters, ruling out a major effect on clustering from sample-specific technical artifacts or of patient-specific phenotypes.

All tSNE-defined clusters encompassed both $\operatorname{IgA}^{+}$and $\operatorname{IgA}^{-} \mathrm{PBs}$, indicating that PBs of different isotypes can be similarly imprinted. However, the ratio of $\operatorname{IgA}^{+}$to $\operatorname{IgA}^{-} \mathrm{PBs}$ differed substantially between clusters (Figure 2D) and also disease class (as shown in Figure 1B). The ratio ranged from $0.3 \operatorname{IgA}^{+}$to $\mathrm{IgA}^{-} \mathrm{PBs}$ in cluster 24 , to $3.1 \mathrm{IgA}^{+}$to $\mathrm{IgA}^{-} \mathrm{PBs}$ in cluster 30 . Logistic regressions of $\operatorname{IgA}^{+}$to $\operatorname{IgA}^{-}$ratios for cluster-patient class, or for cluster or patient class individually showed that TPs (cluster ID) and patient class are independently predictive of $\mathrm{PB} \operatorname{IgA}$ expression $(P \approx 0)$. Thus, even when the correlation of disease class and TP (cluster) is considered, imprinted TPs remain strongly correlated with and predictive of PB isotype expression. The results suggest that environmental factors that imprint TPs and control isotype switching may share some commonality.

Association of PB TPs with sites of immune activity and disease symptoms. We next evaluated the representation of PBs within each cluster in individual patients with localized symptoms or after influenza vaccination. We limited this analysis to samples with an elevated PB response (compared with healthy donors), defined arbitrarily as having a PB frequency of greater than $0.25 \%$ of blood lymphocytes (see Figure $1 \mathrm{~A}$ ). Of the 115 study participants enrolled, 44 patient samples (8 GI, 5 UC, 2 CeD, 13 URI, 4 Strep, 7 TIV, and 5 ID-TIV) met the percentage PB cutoff criteria and were included in the dataset. Eight HD samples were included for comparison; thus, 52 total samples were used for analysis. The relative number of PBs from each donor falling within each cluster is displayed as a heatmap in Figure 3A. Clusters are ordered in the heatmap by unsupervised hierarchical clustering; the figure shows that the distribution of cells in the 99 clusters is related to the disease class.

Correlation of individual patients by their cluster profiles (the percentage of PBs per cluster within each patient, for all clusters) is illustrated in Figure 3B. The representation of cluster-defined PB subsets is more often akin in patients with similar clinical classification or symptoms, and often quite distinct between patients in different classes. Together, the results demonstrate that a common array of relatively distinct TPs can be imprinted upon PBs in most or all individuals, but suggest that the representation of PBs in these different TPs is a function, at least in part, of recent tissue-specific immune activity.

Enrichment of $t S N E$-defined PB phenotypes with patient class. To determine whether any tSNE-defined PB TPs (clusters) were significantly enriched in immunized or sick patients of a given class, we applied ANOVA to a correspondence table of the percentage of each patient's PBs in 98 of the 99 tSNE-defined clusters. One cluster, selected as the cluster with the least correlation with patient class, was excluded so that values for the 
A

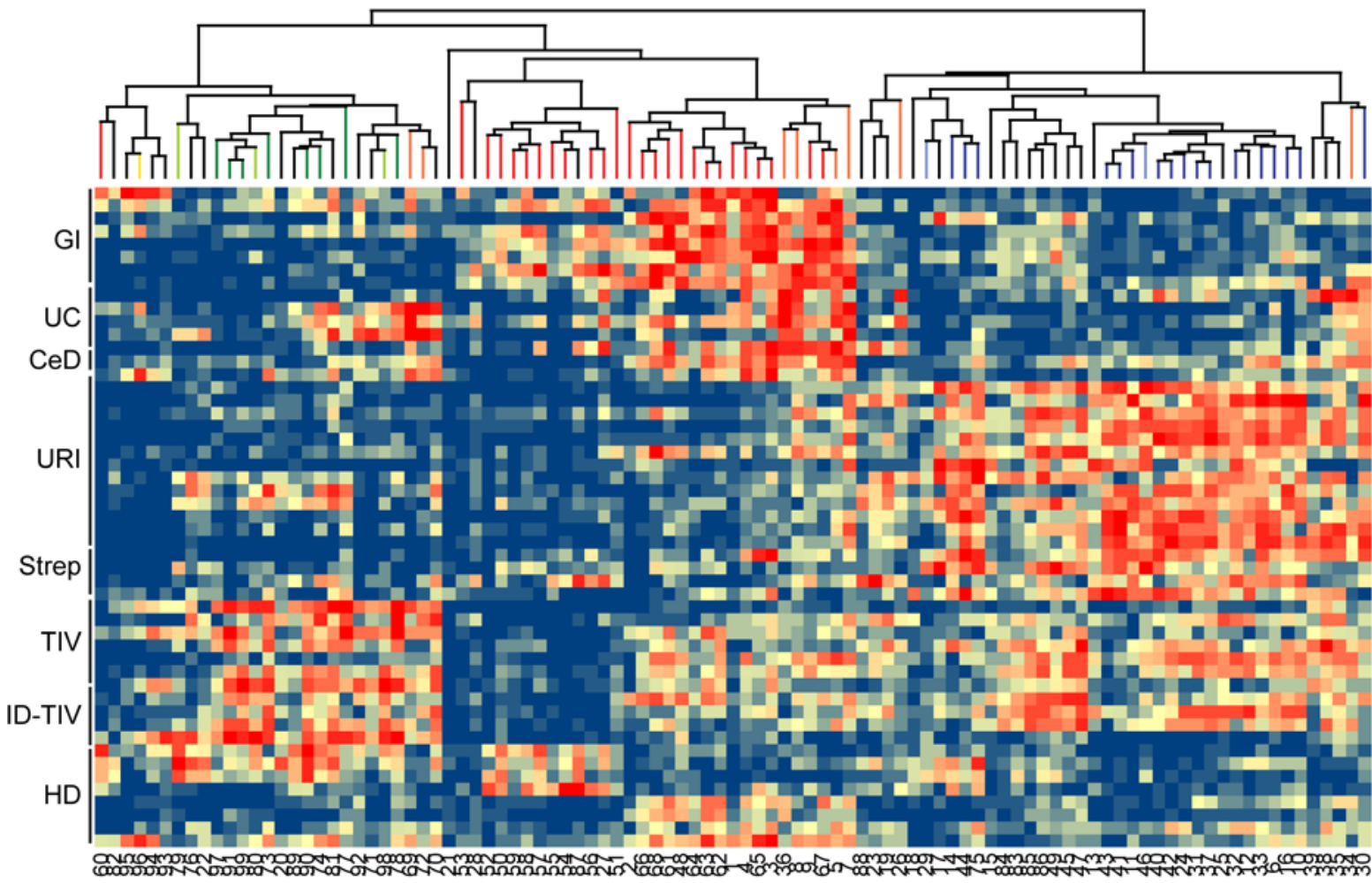

0

10

B

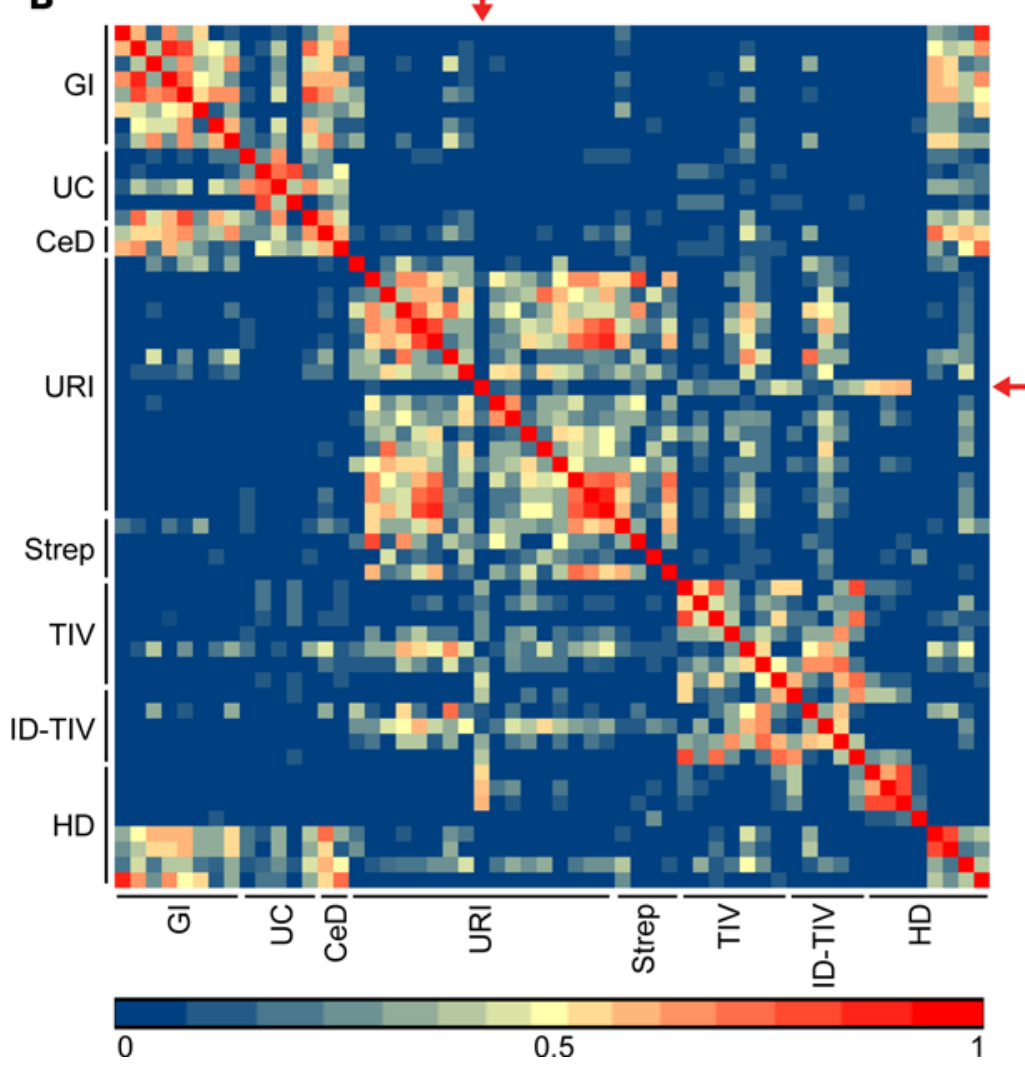

Figure 3. Distribution of plasmablasts among

tSNE-defined clusters. (A) Heatmap illustrating the number of plasmablasts (PBs) in each cluster for each individual patient $(n=52)$. Barnes-Hut t-distributed stochastic neighbor embedding ( $\mathrm{tSNE}$ )-defined clusters are indicated on the $x$ axis and ordered by unsupervised hierarchical clustering via Pearson's correlation of cluster PB profiles (profiles of PB frequencies in the cluster for all patients). Colored branches of the dendrogram indicate clusters that are statistically enriched for a given patient class as indicated (ANOVA). (B) Pairwise Pearson's (linear) correlation of patient PB cluster profiles (percentage of each patient's PBs in the tSNE-defined clusters). The tSNE cluster profile of patient URI_NO23, indicated by the red arrow, was an outlier compared with other URI patients, and thus excluded from subsequent analyses. GI, gastrointestinal infection; UC, ulcerative colitis; CeD, celiac disease; URI, upper respiratory infection; Strep, streptococcal infection; TIV, intramuscular trivalent influenza vaccination; ID-TIV, intradermal TIV; HD, healthy donor. 
98 analyzed clusters would be independent variables. A single patient was excluded (URI_N023, indicated by an arrow in Figure 3B) that had symptoms restricted to URI (sore throat, congestion, productive cough, without fever) but whose tSNE cluster profile was an outlier compared with those of all other URI patients ( $P<0.01$, Q test based on comparisons of each URI patient's mean correlation with all others).

ANOVA revealed significant correlations of tSNE-defined clusters or sets of clusters with patient class. Significantly correlated clusters are highlighted by colored branches of the dendrogram in Figure 3A. Figure 4 illustrates the distribution of significant clusters in tSNE maps, and the phenotype of PBs in the enriched clusters, for patients of different classes.

PBs in clusters enriched in patients with GI symptoms had high expression of $\alpha 4 \beta 7$ and, with few exceptions, high CCR10 expression as well. $\alpha 4 \beta 7$ and CCR10 are known to mediate PB homing to the intestines in mice $(6,10,11)$. GI patient-enriched clusters were distinguished from each other by differences in expression of other receptors. For example, PBs in some but not other clusters had high expression of the small intestinal homing receptor CCR9, potentially reflecting imprinting during immune responses within the small intestine. CD62L was expressed moderately by PBs within most GI-enriched clusters, but P-selectin-ligand binding and CLA were weak or, more often, absent. CD49f was generally absent or only weakly expressed by PBs in most GI-associated clusters.

Even though celiac disease is considered to be a T cell-mediated condition and celiac patients had low percentages of PBs compared with other diseases (Figure 1), 4 clusters were significantly enriched in the $\mathrm{CeD}$ class compared with others as a whole (Figure 4C). These clusters comprised PBs with high expression of CCR9 and $\alpha 4 \beta 7$, known trafficking receptors for the small intestine. Three of the 4 clusters had PBs with high CCR10 expression. CD62L and CLA were relatively poorly expressed, and P-selectin-ligand binding and CD49f reactivity were absent to very low. Enrichment in cells with similar TP signatures was confirmed in 4 independent celiac patients (see Supplemental Figure 2 and Figure 3A).

UC patients showed enrichment for clusters of PBs characterized by high $\alpha 4 \beta 7$ expression without the small intestinal trafficking receptor CCR9. CCR10 ranged from high to low, CLA was generally low or absent, but several enriched clusters contained PBs with high P-selectin binding and, surprisingly, CD49f expression. $\mathrm{PBs}$ were $\mathrm{CD} 62 \mathrm{~L}$ positive, expressing the receptor at intermediate levels, comparable to that of CD62L $\mathrm{L}^{+} \mathrm{PBs}$ in GI-enriched clusters but lower than on PBs in many URI-enriched populations (see below). The lack of CCR9 on enriched PBs in UC is consistent with selective imprinting of CCR9 in response to small intestinal immune challenge (i.e., in GI infection and CeD in Figure 4, A and C), whereas $\alpha 4 \beta 7$ and CCR10 are induced on B cells responding to challenge in either the large or small intestines. The induction of P-selectin-binding PBs in the UC cohort may reflect tissue-selective imprinting, but could also be a function of a strong acute inflammatory response that is seen during severe recurrent disease, as evident in the patients recruited for the current study. Our UC patients were also younger as a group than other patients studied (Supplemental Figure 4), so age-associated differences in PB imprinting cannot be excluded.

URI was associated with enrichment in clusters of PBs with poor expression of CCR 9 and $\alpha 4 \beta 7$, high expression of CD49f and CD62L, and intermediate, variable expression of CCR10. Several clusters had PBs with high P-selectin binding, and a subset of these shared high expression of CLA. Low or absent $\alpha 4 \beta 7$ on PBs correlates with high expression of the vascular cell adhesion molecule 1 (VCAM1) receptor, $\alpha 4 \beta 1$ (see insert histogram, Figure 4D). The expression of the 2 integrins is inversely related on circulating $\mathrm{PB}$ populations, as shown in Figure 4. VCAM1 mediates naive B and T lymphocyte homing to bronchusassociated lymphoid tissues (21) and T cell homing to the lungs in mice (22), and CD62L is implicated in $\mathrm{T}$ cell-mediated bronchopulmonary responses in asthma models in sheep (23). The results support a role for VCAM1 and selectin-mediated interactions, particularly L-selectin (CD62L), in human PB trafficking during respiratory tract immune challenge.

Four patients with total PBs greater than $0.25 \%$ of lymphocytes had symptoms limited to severe sore throat with a medically confirmed diagnosis of streptococcal infection. The enriched PBs showed high expression of CLA in comparison with the other patients as a whole. Clusters enriched at $P$ less than 0.005 were populations predominantly characterized by PBs with intermediate CCR10 expression and P-selectin binding, with subsets of P-selectin-ligand ${ }^{\text {hi }}$ cells in some clusters. The most populous clusters had high expression of CD62L as well. In direct comparisons with URI patients (many of whom also had throat symptoms), the culture-confirmed strep throat samples were enriched in the CLA ${ }^{\text {hi }}$ cluster $46(P<0.05)$, and in the relatively poorly populated CCR9 ${ }^{+} \alpha 4 \beta 7^{+}$clusters 54 and $55(P<0.005)$. Enrichment in the latter clusters may suggest stimulation of the proximal intestines by swallowed antigen. Analysis of a separate 


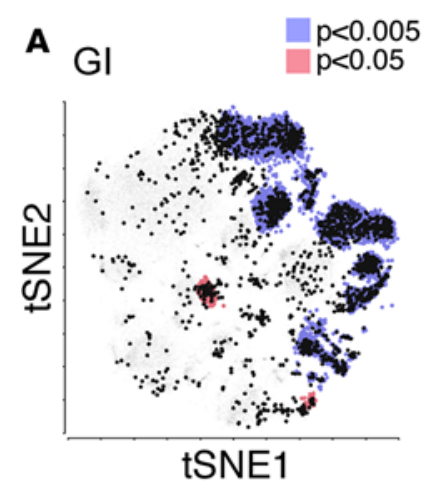

B UC

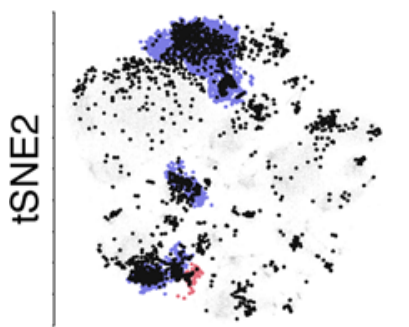

\section{c CeD tSNE1}

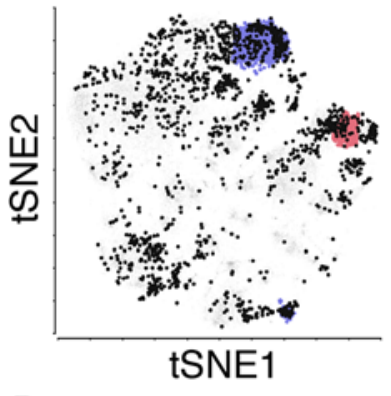

D URI

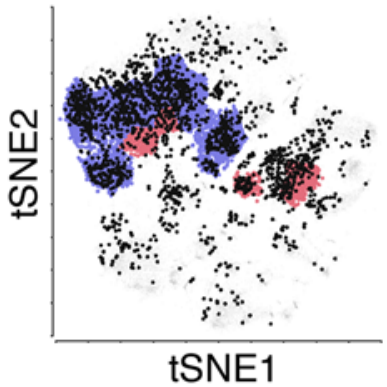

E Strep
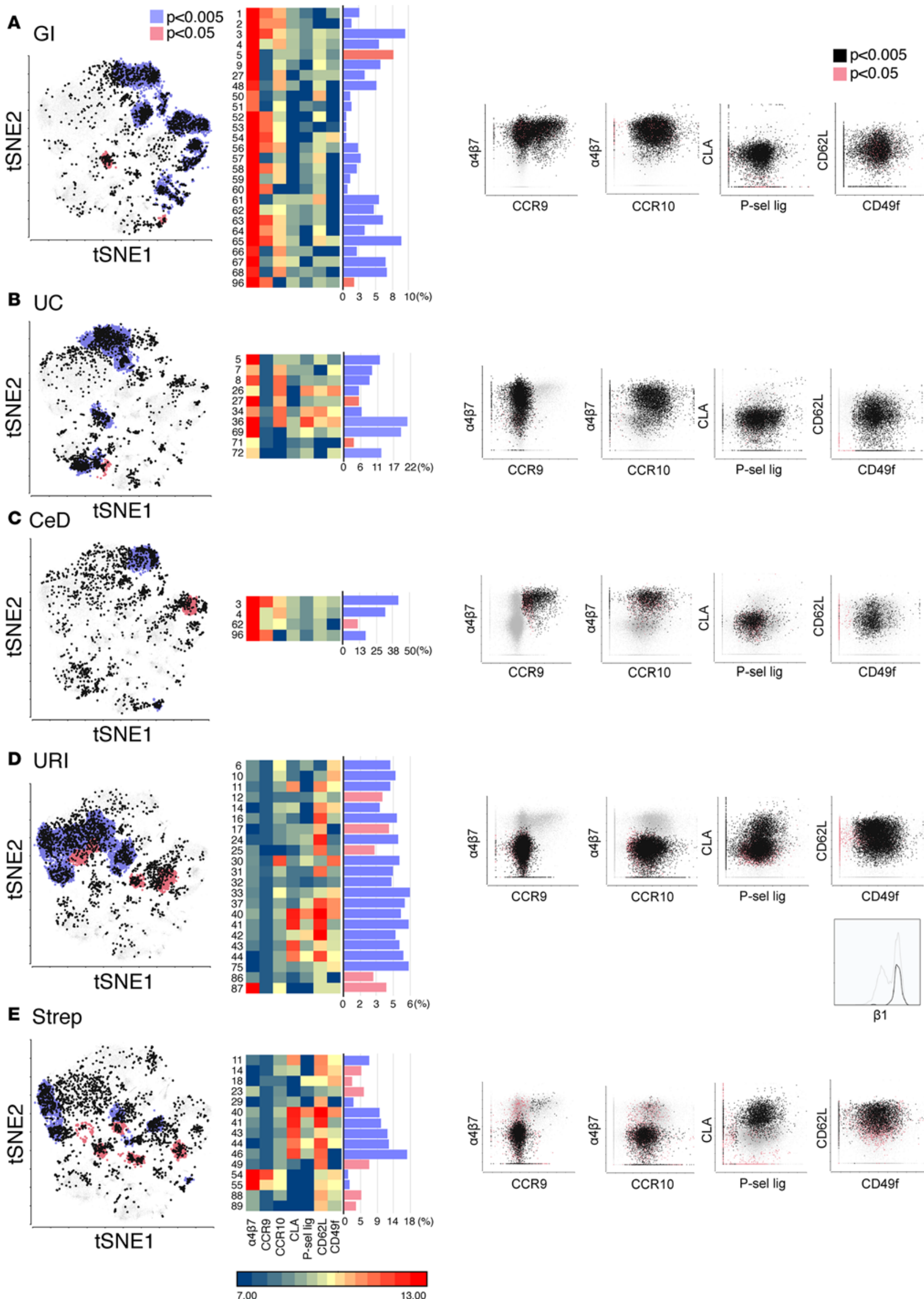
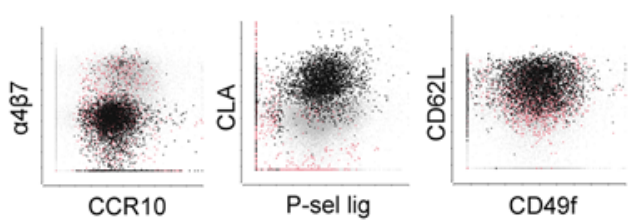

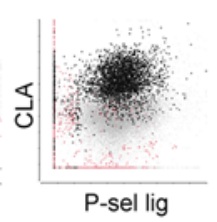


Figure 4. Imprinted trafficking programs of plasmablasts in statistically enriched (disease- or inflammatory site-associated) clusters. The phenotype of plasmablasts (PBs) in enriched clusters is shown for patients with (A) gastrointestinal infection (CI) symptoms, (B) ulcerative colitis (UC), (C) celiac disease (CeD), (D) upper respiratory infection (URI) symptoms, and (E) streptococcal infection (Strep). In each panel A-E, the Barnes-Hut t-distributed stochastic neighbor embedding (tSNE) map shows 2,000 PBs randomly selected from the patient class; statistically enriched clusters are colored. The heatmap shows trafficking receptor expression (mean fluorescence intensity) of PBs within each enriched cluster; an associated bar graph indicates the relative abundance of PBs in the enriched clusters. Conventional $2 \times 2$ flow cytometry plots of PBs from enriched phenotypes are displayed. For URI patients, a histogram insert illustrates staining for integrin $\beta 1$ on $\alpha 4 \beta 7^{-} \mathrm{PBs}$, consistent with high $\alpha 4 \beta 1$ integrin expression (representative of $n=4$ ). Cells (and bars in the histogram) from statistically enriched clusters are colored by $P$ value as indicated (ANOVA, see Methods).

cohort of 3 patients with strep throat showed imprinting of similar phenotypes including TPs with high CLA and CD62L and low $\alpha 4 \beta 7$ (Supplemental Figure 3B). The results suggest a role for CLA and potentially CD62L in PB recruitment to the inflamed oral cavity.

Phenotype of influenza-specific PBs induced by intramuscular or intradermal TIV. Vaccination allows us to probe the $\mathrm{PB}$ response to a known antigenic stimulus in a controlled tissue site. We evaluated PBs in 41 patients following TIV or ID-TIV. Of these patients, only 7 after TIV and 5 after ID-TIV had blood PB levels greater than $0.25 \%$, making them suitable for further evaluation. We also assessed the PB response in subjects receiving Flumist, the influenza vaccine administered intranasally; however, Flumist did not elicit a $\mathrm{PB}$ response above that of HDs and thus, these subjects were not included in the study (data not shown).

Overall, these 2 types of vaccination imprint similar trafficking programs. Six out of 12 clusters are shared between both TIV- and ID-TIV-enriched phenotypes. In comparison with other samples as a group, $\mathrm{PBs}$ in both types of vaccine recipients were enriched in clusters of PBs lacking the intestinal and mucosal trafficking receptors $\alpha 4 \beta 7$, CCR9, and CCR10, but in most cases PBs expressed CD62L at high levels (Figure 5A). P-selectin binding was variable but high in many clusters, especially after TIV immunization; in direct comparison between the vaccinated groups, PBs after TIV were significantly enriched $(P<0.05)$ in cluster 39, 77, and 78, each of which is characterized by very high P-selectin binding, whereas ID-TIV PBs were enriched in cluster 80, characterized by CD62L expression but the lack of P-selectin binding and expression of other trafficking receptors. Similarity between ID-TIV and TIV responses might be due to the fact that the antigens are destined for the same lymph nodes and prime the PBs under a similar microenvironmental condition. Most importantly, the complete absence of CCR10 expression on PBs in most TIV- and ID-TIV-enriched clusters suggests that CCR10 is imprinted on PB in response to immune stimuli at mucosal but not subcutaneous sites. However, the results do not exclude a difference related to vaccine versus infectious or other immune stimuli.

As shown in Figures 1 and 5, PBs in vaccinees were enriched in $\operatorname{IgA}^{-}\left(\operatorname{IgG}^{+}\right)$cells and in $\alpha 4 \beta 7^{-}$and CCR10- subsets as compared with other patient groups. To determine whether influenza-specific PBs were similarly enriched in these populations, we assayed influenza antigen (flu-Ag)-specific ASCs among sorted $\alpha 4 \beta 7^{+}$and $\alpha 4 \beta 7$ subsets of $\operatorname{IgA}^{+}$and $\operatorname{IgA}^{-}\left(\operatorname{IgG}^{+}\right)$PBs from 4 patients, and among separately sorted $\mathrm{IgA}^{-}\left(\mathrm{IgG}^{+}\right) \alpha 4 \beta 7^{-} \mathrm{CCR} 10^{+}$versus $\alpha 4 \beta 7^{-} \mathrm{CCR} 10^{-}$subsets from 5 patients by enzyme-linked immunospot (ELISPOT) assay. Inactivated influenza vaccine (Fluzone) was used to detect flu-Ag-specific ASCs. Figure 5C displays the fraction of flu-Ag-specific ASCs comprised within each phenotypically defined subset (the percentage of all flu-Ag-specific PBs that fell within each sorted subset was calculated as the percentage ELISPOT $^{+}$ASCs within sorted cells of that subset, multiplied by the percentage representation of that subset among all PBs, divided by the percentage of separately sorted total PBs that were ELISPOT ${ }^{+}$). Although cells producing flu-Ag-specific antibody were found among all sorted subsets in at least some patients, they were predominantly IgG-producing $\alpha 4 \beta 7^{-}$(presumably $\alpha 4 \beta 1^{\mathrm{hi}}$ ) and CCR $10^{-}$. We conclude that intramuscular or intradermal immunization with conventional flu vaccine leads to the induction of flu-Ag-specific IgG ASCs that lack mucosal trafficking programs, consistent with the phenotype of enriched PB clusters in these patient classes. However, minor subsets of flu-Ag-specific cells after vaccination may express trafficking receptor profiles consistent with post-URI or post-GI PB phenotypes.

Extent of enrichment of PBs expressing tissue-targeting trafficking programs. The results define characteristic phenotypes associated with PBs imprinted in the context of localized acute infection and inflammatory symptoms. To determine the extent of enrichment in the imprinted phenotypes within patients, we calculated the percentage of each patient's PBs displaying identified patient class-associated TPs or profiles, and normalized these values to the average percentage of PBs from each disease class within the same gated phenotype. The results were calculated separately for $\operatorname{IgA}^{+}$and $\operatorname{IgA}^{-} \mathrm{PB}$ subsets, and are presented as fold enrichment in Figure 6. The mean percentage of PBs with enriched phenotypes ranged from $16 \%$ in $\mathrm{CeD}$ to $88 \%$ in UC. 
A TIV $\quad$ p $<0.005$
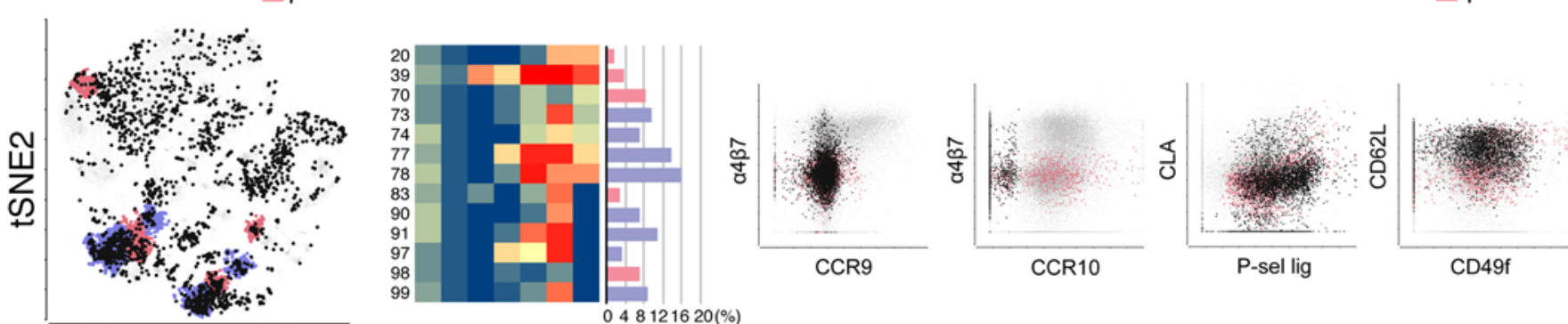

tSNE1

B ID-TIV

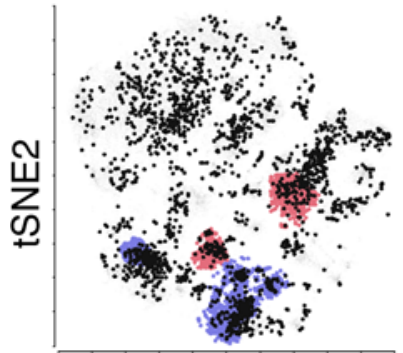

tSNE1
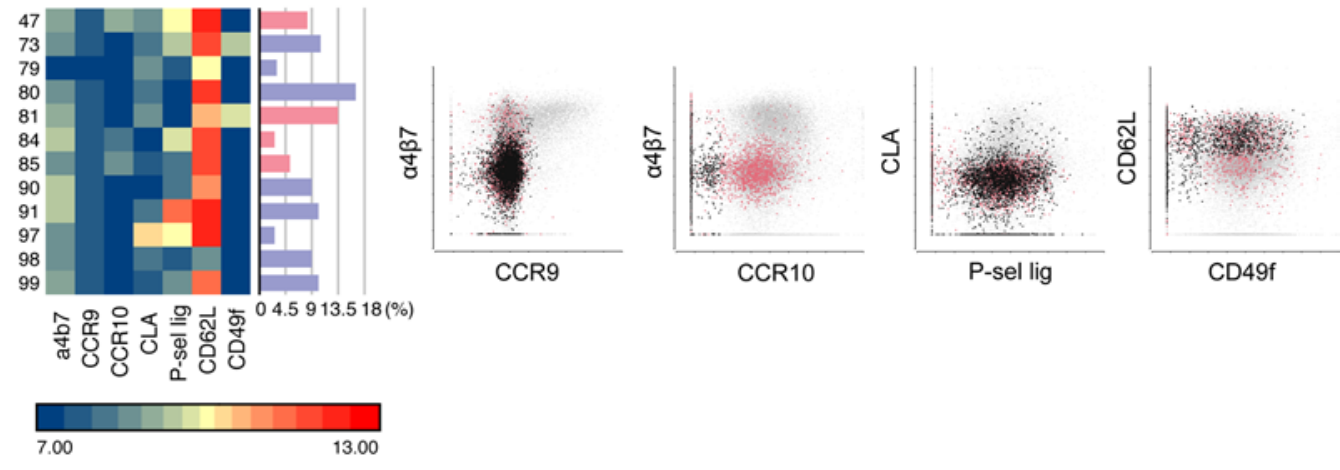

C

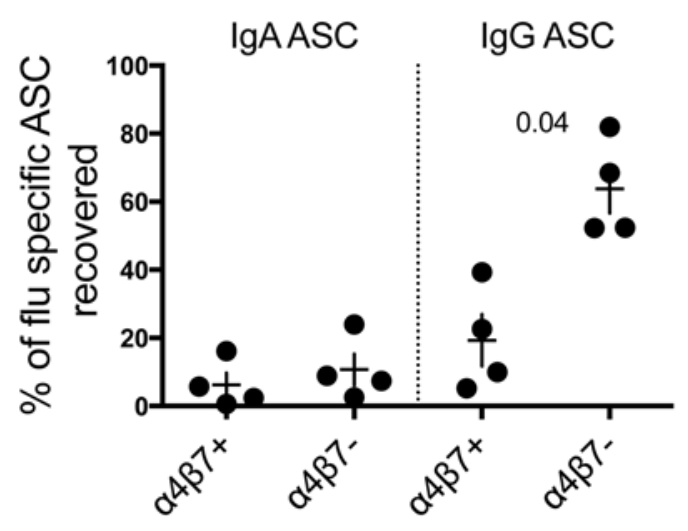

D

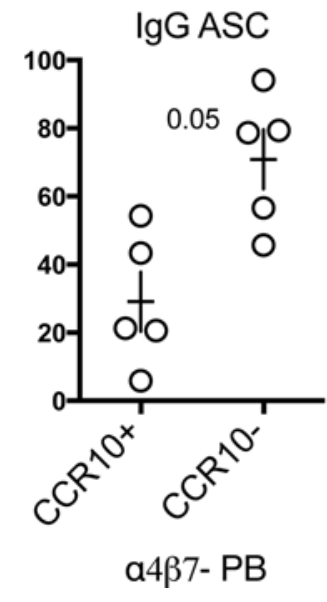

Figure 5. Intramuscular and intradermal influenza vaccinations induce influenza-antigen-specific IgG plasmablasts that lack mucosal trafficking programs. The phenotype of plasmablasts (PBs) in enriched clusters is shown for patients receiving $(\mathbf{A})$ intramuscular trivalent influenza vaccination (TIV, $n=7$ ) or (B) intradermal TIV (ID-TIV, $n=5$ ). tSNE, Barnes-Hut t-distributed stochastic neighbor embedding. See the Figure 4 legend for explanation of figure panels and statistics. (C) $\alpha 4 \beta 7$ expression by flu-Ag-specific antibody-secreting cells (ASCs). Shown is the percentage of the total recovered flu-Ag-specific ASCs among sorted $\alpha 4 \beta 7$-positive or -negative fractions of IgA-secreting cells (IgA ASCs) or IgG-secreting cells (IgC ASCs). Each data point is from an individual patient $(n=4)$. (D) The fraction of flu-Ag-specific IgC ASCs among $\alpha 4 \beta 7^{-}$IgA- PBs that sorted with CCR10 ${ }^{+}$or CCR10subsets $(n=5)$. In $\mathbf{C}$ and $\mathbf{D}, P$ values were determined by paired-ratio, 1-tailed $t$ test.

We similarly evaluated IgA $\mathrm{A}^{+}$memory B cells, identified in our staining panel as $\mathrm{CD} 19^{+}$among CD4- $\operatorname{IgA}{ }^{+} \mathrm{CD} 38^{\text {int }}$ cells. The enriched disease-specific phenotypes observed in PBs were evaluated within the IgA $\mathrm{A}^{+}$memory subset. Memory B cells were represented within each of the tissue-specific phenotype classes, but unlike PBs, memory cells were not significantly enriched in any diseasespecific phenotypes. 
A UC phenotype

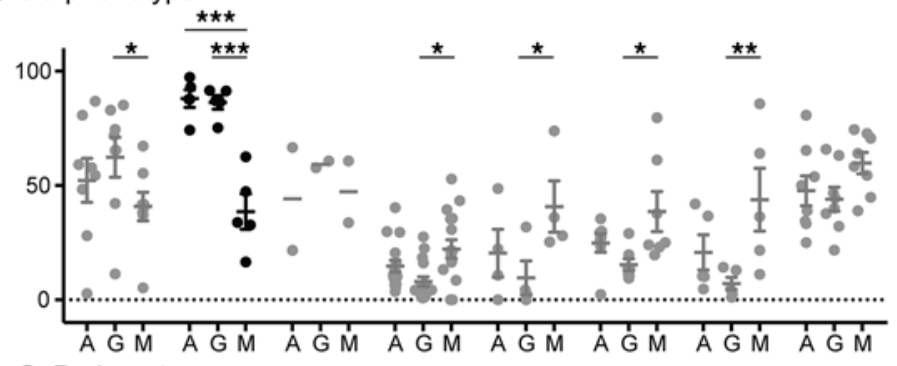

B CeD phenotype

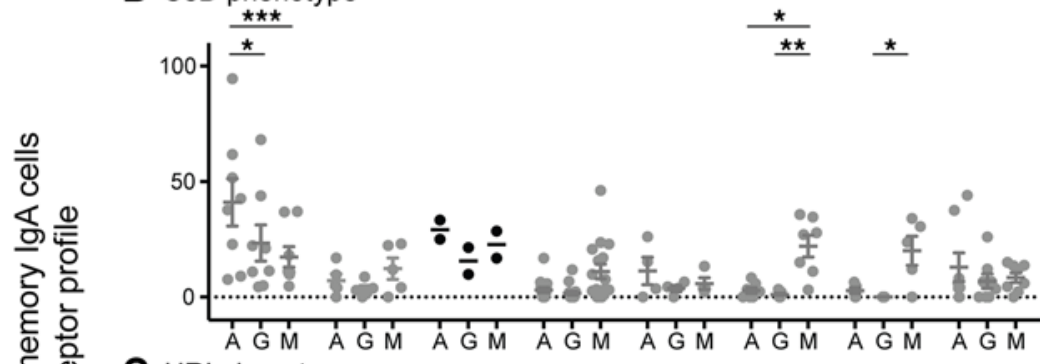

C URI phenotype

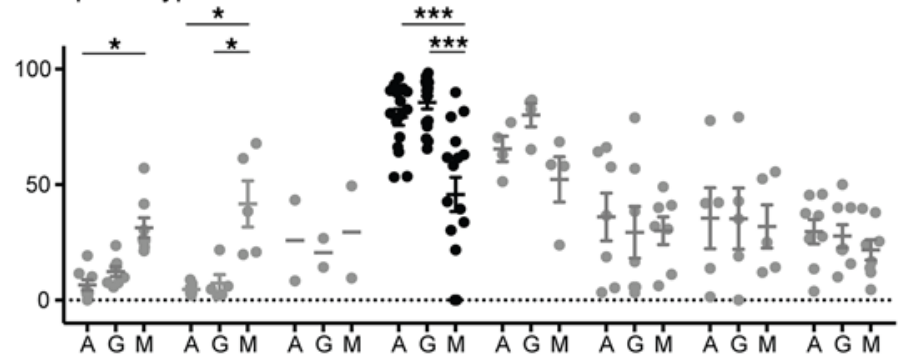

D Strep phenotype

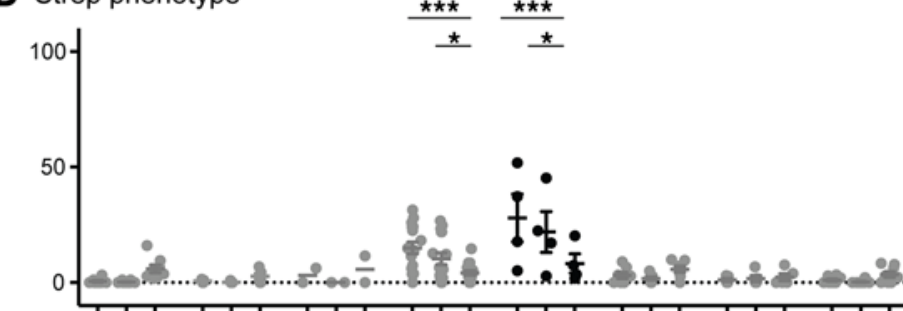

$\dot{A} \dot{G} \dot{M} \quad \dot{A} G \dot{M} \quad \dot{A} G \dot{M} \quad \dot{A} G \dot{M} \quad \dot{A} G \dot{M} \quad \dot{A} G \dot{M} \quad \dot{A} G \dot{M} \quad \dot{A} G \dot{M}$

E TIV and ID-TIV phenotype

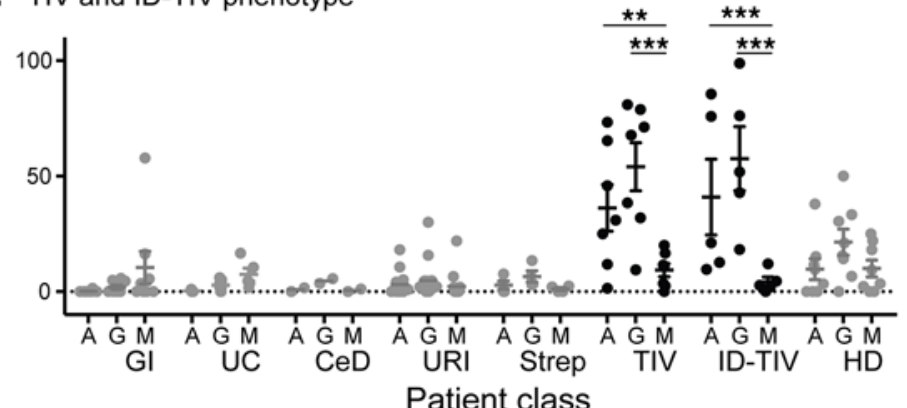

Patient class
Figure 6. Representation of tissue-specific trafficking programs on plasmablasts (PBs) and memory $B$ cells in patients. Each graph presents the percentage of patients' cells imprinted with the indicated tissueselective trafficking programs (TPs). Graphs of percentage of indicated cell types that display (A) ulcerative colitis (UC), (B) celiac disease (CeD), (C) upper respiratory infection (URI), (D) streptococcal infection (Strep), and (E) trivalent influenza vaccination (TIV)-associated and intradermal TIV (ID-TIV)-associated TPs. Patient samples, including healthy donors (HDs) and patients with gastrointestinal infection (GI), are grouped according to their disease class (indicated on the $x$ axis). IgA ${ }^{+}$ $\mathrm{PBs}$ (indicated by open " $\mathrm{A}$ " on the abscissa), IgA- PBs (G) or IgA $A^{+}$memory B cells (M) are evaluated separately. Each data point is from an individual patient $(n=52)$, and represents the percentage of the indicated cell type that displays a pattern of trafficking receptors characteristic of PBs in statistically enriched TP clusters $(P<0.005$, from Figure 4$)$ in the disease class evaluated in the graph. ${ }^{*} P<0.05,{ }^{* *} P<0.005,{ }^{* *} P<0.0005$ by 2-way ANOVA.

\section{Discussion}

We have used single-cell analysis of trafficking receptor expression to define combinations of adhesion molecules and chemoattractant receptors imprinted on $\mathrm{PBs}$ in patients 7 days after influenza vaccination or 7 to 9 days after acute inflammatory symptoms or during acute exacerbations of tissue-specific autoimmune disease. Our results show that PB TPs correlate with sites of immune challenge, and we define characteristic TPs associated with the small intestine, colon, upper respiratory tract, and throat. Intramuscular and intradermal administration of the influenza vaccine induces a distinct TP from those imprinted by mucosal immune responses. These results provide an experimental framework for identifying trafficking receptors and their unique combinations that determine lymphocyte homing during the dissemination of active immune responses, and define a set of parameters that will permit blood monitoring and analysis of immune activity occurring within distinct tissues of the body.

We applied the Barnes-Hut variant of tSNE for dimensionality reduction, and a variant of k-means clustering to define clusters based on trafficking receptor profiles. K-means clustering defines clusters even within a uniform or uni-

modally distributed field of cells; this feature is critical for biological analysis, since many receptors on whole populations of cells, including some of the trafficking receptors studied here, can be expressed in a unimodal fashion and yet vary in expression between individual cells by orders of magnitude. Statistical analysis confirms that in these cases, PBs displayed characteristically high or low levels of receptor expression in patients with different symptoms. Among the 99 trafficking receptor-defined clusters used in our analysis, even the most similar pairs of clusters (defined by Pearson correlation of their mean trafficking receptor expression profiles) differed by 2 - to 3 -fold in 2 or more parameters, or up to 4 -fold 
in at least one parameter. Such differences are expected to be physiologically significant in the context of the sequential engagement of trafficking receptors during $\mathrm{PB}$ recruitment.

In rodents and humans, IgA ASCs predominate in mucosal lymphoid tissues in the gut and respiratory tract, whereas IgG ASCs predominate in systemic (nonmucosal) sites of tissue inflammation $(6,24)$. We observed significant differences in induction of $\operatorname{IgA}^{+}$versus $\operatorname{IgA}^{-}$PBs in patients, correlating with disease class and with cluster (trafficking profiles). As expected, $\operatorname{IgA}^{+} \mathrm{PBs}$ are most highly enriched after intestinal infection, whereas $\mathrm{IgA}^{-} \mathrm{PBs}$ (mostly $\mathrm{IgG}^{+}$in the recall response) predominated after influenza vaccination. $\operatorname{IgA}^{+}$and $\operatorname{IgA}^{-}$PBs also differed in relative proportion in different $\mathrm{TP}$ clusters, though all clusters comprised cells of both isotypes. More surprising than these differences, however, was the overall similarity in enriched TPs of $\mathrm{IgA}^{+}$versus $\mathrm{IgA}^{-} \mathrm{PB}$ subsets within each patient. Studies in the mouse had led to the expectation that IgG-expressing PBs would largely lack intestinal or mucosal trafficking receptors (6). Mouse studies, however, focused primarily on tissue-resident plasma cells rather than blood PBs in the active immune response. The similarity of TPs on $\mathrm{IgA}^{+}$and $\mathrm{IgG}^{+} \mathrm{PBs}$ suggests that tissue targeting during the immune response may be imprinted through common mechanisms on the PB subsets, and that other factors may contribute to the ultimate predominance of IgA-producing ASCs in mucosal tissues and IgGproducing ASCs in systemic sites. There may be differences in survival or retention of $\operatorname{IgA}^{+}$versus $\operatorname{IgG}^{+}$ $\mathrm{PBs}$ in different tissues. Interestingly, Kuklin et al. showed that gut-homing B cells in IgA knockout mice are as protective as in wild type, indicating that IgA deficiency can be compensated by other isotypes (8). In contrast, mice deficient in $\beta 7$ integrin showed decreased protection. Thus, migration of $\mathrm{PBs}$ to the small intestine may allow $\mathrm{IgG}^{+}$as well as $\mathrm{IgA}^{+} \mathrm{PBs}$ to contribute to protective mucosal immune responses.

In general, the TPs imprinted on PBs correlate well with previously known patterns of vascular/tissue-selective adhesion molecule and chemoattractant receptor expression. $\alpha 4 \beta 7$, imprinted in patients with small intestinal and colonic inflammation, mediates lymphocyte rolling and activation-dependent adhesion to the mucosal vascular addressin MAdCAM1 $(25,26)$. MAdCAM1 decorates venules in Peyer's patches and throughout the intestinal lamina propria. CCR9, imprinted on PBs in patients with known (in celiac disease) or presumed small intestinal involvement, is the adhesion-triggering and chemoattractant receptor for CCL25, a chemokine that is selectively expressed by small intestinal epithelium (27). CCR10, imprinted on most or all PBs responding to mucosal immune challenge, mediates adhesion and chemotaxis to CCL28, a common mucosal chemokine expressed by bronchial, salivary, oral, and intestinal epithelium (10, 12, 27). CCL28 is more highly expressed in colon than small intestinal sites. In colitis, where disease is restricted to the large intestine and involves both chronic immune activity and innate acute/subacute inflammation, imprinted PBs were $\alpha 4 \beta 7^{+} \mathrm{CCR} 10^{+}$and often P-selectin-ligand ${ }^{\text {hi }}$ (and CLA ${ }^{\text {lo }} \mathrm{CD} 62 \mathrm{~L}^{\text {int }} \mathrm{CD} 49 \mathrm{f}^{\mathrm{fnt}}$ ) but lacked CCR9 expression. P-selectin has been implicated in colitis in chemically induced models associated with strong innate responses (28). A high P-selectin-binding subset was also present in other disease classes studied, particularly in patients with strep throat or after vaccination. P-selectin is classically induced or translocated from constitutive stores to the cell surface of endothelial cells at sites of acute inflammation. P-selectin binding ability may be imprinted by environmental and inflammatory factors associated with acute inflammation, rather than tissue-specific factors. High $\alpha 4 \beta 1$ and CD62L expression characterizes PBs imprinted by respiratory tract challenge. CD62L mediates tethering and rolling on endothelial Sialyl Lewis X-related carbohydrate ligands, including the peripheral lymph node addressin PNAd $(29,30)$. PNAd decorates high endothelial venules in bronchus-associated lymphoid tissues, vessels that also express the $\alpha 4 \beta 1$ ligand VCAM1 (21). Both CD62L and $\alpha 4 \beta 1$ have been implicated in pathogenic immune recruitment to airways (31); inhibition of CD62L prevents the induction of airway hypersensitivity in a sheep model of asthma (23) and $\alpha 4$ antagonism inhibits $\mathrm{T}$ cell and eosinophil recruitment to the airway in a mouse model of asthma $(32,33)$. In combination with CCR10, CD62L and $\alpha 4 \beta 1$ may provide efficient targeting of PBs to bronchial and perivascular connective tissue in pulmonary sites. PBs in strep throat patients were enriched in cells with high expression of CLA, a Sialyl Lewis X-related carbohydrate ligand for the vascular adhesion receptor CD62E (E-selectin) (34). E-selectin is constitutively expressed by venules of the oral mucosa $(35,36)$ as in the skin (but not intestines) $(34,37)$. It is further upregulated in the inflamed oral mucosae, as is VCAM1. Although the mechanisms of lymphocyte homing to the oral mucosae have not been probed experimentally, CLA mediates lymphocyte homing to skin via interaction with vascular E-selectin $(34,37)$. Our results suggest that the stratified squamous epithelium of the oral mucosa may share common CLA-imprinting mechanisms with skin keratinocytes. Like other mucosally imprinted PBs, those induced by throat infection are CCR $10^{+}$as well. CLA in combination with $\alpha 4 \beta 1$ and CCR10 may mediate efficient recruitment to the inflamed oral mucosae. 
Our results confirm and extend previous studies of PB trafficking receptors in patients. Czerkinsky et al. used antibody-coated-bead enrichment to demonstrate enriched $\alpha 4 \beta 7$ expression in antigen-specific PB responding to oral immunization with cholera vaccine (38). Using flow cytometry, Kantele et al. observed a4ß7 expression by ASCs after oral vaccination with Salmonella typhi, whereas CD62L was enriched after systemic (intramuscular) immunization with the same antigen (15). Studies of rotavirus, which selectively infects small intestinal epithelium in patients, found that rotavirus antigen-specific $\mathrm{IgA}^{+} \mathrm{PBs}$ display high levels of CCR9, CCR10, and $\alpha 4 \beta 7$ (1), consistent with the patterns of imprinting in celiac disease as shown here and in a report by Tarlton et al. (39).

Subsets of memory B cell express the trafficking receptors studied here (40) and display them in combinations consistent with enriched TPs defined through our PB analyses. In general, the representation of memory B cells with different tissue-targeted phenotypes did not correlate or vary substantially with patient/disease class. Long-lived circulating memory cells may even be depleted from the blood by recruitment during active immune responses in tissues (39), potentially balanced by new memory cell generation which would likely follow the initial influx of activated lymphocytes during resolution of the response. In addition to the trafficking receptors we evaluated, memory B cells express CCR6 (41). The CCR6 ligand CCL20 is expressed in lymphoid tissues and sites of inflammation, and is induced in inflamed or infected epithelium $(42,43)$. Thus, memory B cells may be recruited through tissueselective TPs similar to those used by PBs, but also through CCL20-dependent and other mechanisms to epithelial sites of inflammation.

Vaccination with TIV imprinted PBs with an $\alpha 4 \beta 1^{+}$( $\beta 7$ negative) phenotype that displayed P-selectin binding ability, were generally CD62L positive and high, but notably lacked expression of the mucosal chemokine receptor CCR10. The few PBs that were CCR10 $0^{+}$resembled PBs induced during an upper respiratory infection. Our ELISPOT analysis of sorted PBs after influenza vaccination confirmed that $\mathrm{PBs}$ expressing the enriched trafficking programs after vaccination comprised the flu-Ag-specific ASC population in immunized patients' blood. The lack of CCR10 on vaccine-induced PBs contrasts with CCR10 expression by the vast majority of both $\mathrm{IgA}^{+}$and $\mathrm{IgG}^{+} \mathrm{PBs}$ after each of the mucosal immune challenges and in healthy donors. Imprinting of CCR10 on PBs may require factors restricted to mucosal tissues or their draining lymph nodes. The results suggest that intramuscular or intradermal vaccination may be relatively inefficient at generating PBs capable of immediate trafficking to mucosal sites. It is likely that memory cells induced by such vaccination, however, can give rise to efficiently targeted PBs in response to subsequent pathogen challenge.

Expression of integrin $\alpha 6$ (CD49f) varied with cluster and disease like the known trafficking receptors. It was most highly expressed by PBs in URI and Strep, and was absent in most clusters enriched in CeD. $\alpha 6 \beta 1$ is a receptor for laminins (44), extracellular matrix components comprised of multiple isoforms whose expression varies in different tissues (45). $\alpha 6$ binding to laminins is thought to play an important role in platelet-mediated thrombosis (46). The role of $\alpha 6$ in lymphocyte trafficking, if any, remains to be determined; we have not seen alterations in lymphocyte homing to lymph nodes or Peyer's patches with an anti- $\alpha 6$ antibody that blocks laminin binding (Eugene C. Butcher, unpublished observations).

It will be important to extend studies to other adhesion molecules and chemoattractant receptors that may contribute to PB trafficking. CXCR3, a chemokine receptor associated with trafficking to inflamed sites, is expressed by PBs within each of the trafficking receptor disease-associated phenotypes described here, and may participate in regulating recruitment or environmental positioning independent of tissue specificity, potentially as a function of the nature of inflammatory insult or cytokine environment (Yekyung Seong and Nicole H. Lazarus, unpublished observations). Its expression correlates loosely but significantly with that of P-selectin binding. G protein-coupled receptor 15 (GPR15), which is implicated in $\mathrm{T}$ cell homing to the colon and potentially to the skin (47-49), is also expressed by subsets of PBs within each disease-associated class, without a clear enrichment in or restriction to cells targeted to intestinal sites (Y. Seong and N.H. Lazarus, unpublished observations). As proposed for CXCR3, it may regulate microenvironmental localization or inflammatory insult-selective recruitment of PBs. Examination of expression of these and other receptors in a broad panel of patients will be required to determine the correlates of their imprinting on PB subsets. CCR2 is also expressed on subsets of PBs, whereas antibodies against CCR4, CCR5, CXCR5, CD103, and $\alpha 2$, which stained subsets of T cells, did not stain PBs in our hands (Y. Seong and N.H. Lazarus, unpublished observation). We did not study the expression of CXCR4, a receptor implicated in $\mathrm{PB}$ trafficking to the bone marrow (7). 
Table 1. Disease scores of patients with ulcerative colitis as assessed by the pediatric ulcerative colitis activity index (PUCAI) (50)

\begin{tabular}{lcc}
\hline Patient ID & Disease score & Severity \\
N034 & 75 & Severe \\
N035 & 75 & Severe \\
N036 & 65 & Severe \\
N037 & 75 & Severe \\
N054 & 60 & Moderate
\end{tabular}

In conclusion, we present a framework for discovery of unique signatures of blood lymphocytes activated by and participating in localized immune responses, and we identify patterns of trafficking receptor expression that define PBs responding and targeted to colon, small intestine, throat, respiratory tract, or systemic nonmucosal sites of immune challenge. Similar approaches should allow definition of trafficking signatures of $\mathrm{T}$ cell subsets as well, and may facilitate efforts to noninvasively monitor ongoing immune activity in diverse tissues of the body.

\section{Methods}

\section{Patient cohort}

Patients experiencing symptoms of food poisoning or other acute infectious symptoms such as strep throat or upper respiratory infections were enrolled at the Palo Alto Veterans Affairs Health Care System. Subjects that had received ID-TIV or TIV and were asymptomatic for any acute infectious illnesses were enrolled in this study at Stanford University. The vaccine consisted of inactivated influenza A and B components derived from the H1N1 (A/California/7/09), H3N2 (A/ Perth/16/09) and influenza B (B/Brisbane/60/08) strains. All participants received the same vaccine. All subjects completed a short questionnaire asking about their illnesses' duration and type of symptoms experienced and further grouped into disease classes based upon the symptoms they subjectively reported: GI, URI, or Strep. Peripheral blood samples were obtained 7 days after administration of the influenza vaccination or 7 to 9 days after acute infectious symptom onset. Pediatric UC patients were enrolled in this study during routine office visits by the Pediatric Gastroenterologists' Group at Kaiser Permanente in San Jose, California, USA. The disease activity of each UC patient was assessed using the pediatric ulcerative colitis activity index (PUCAI) (50). The UC patients and their corresponding disease severity are shown in Table 1 . Adult $\mathrm{CeD}$ patients were enrolled through the Department of Gastroenterology at the Stanford University School of Medicine. Disease severity was assessed through the Marsh scoring system and peripheral blood samples were obtained during routine office visits. HDs were selected for this study based on the lack of any acute infectious symptoms or chronic conditions such as general allergies (food or environmental), skin disorders and/or joint pain. All study participants were over the age of 18 , except the pediatric UC patients, and none were pregnant.

\section{Lymphocyte separation from whole blood}

Heparinized peripheral blood (between $10 \mathrm{ml}$ and $40 \mathrm{ml}$ ) was obtained via venipuncture from the different donor populations. Blood samples were processed using Ficoll density gradient centrifugation (Histopaque-1077, Sigma-Aldrich). The resulting interface containing the PBMC layer was extracted, washed twice with HBSS without $\mathrm{Ca}^{++} / \mathrm{Mg}^{++}$(Mediatech), and resuspended in staining buffer (PBS without $\mathrm{Ca}^{++} /$ $\mathrm{Mg}^{++}$supplemented with $2 \%$ bovine calf serum and $0.1 \%$ sodium azide). Cells were incubated with 0.1 $\mu 1$ normal human $\mathrm{AB}$ serum (Sigma-Aldrich) and $0.5 \mu 1$ normal goat serum (Gibco) in $100 \mu 1$ of staining buffer per 1 million PBMCs for 15 minutes on ice prior to staining. Cells isolated from UC patients were blocked overnight at $4^{\circ} \mathrm{C}$ in staining buffer containing human and goat serum.

\section{Monoclonal antibodies for flow cytometric phenotypic analysis}

Primary unconjugated antibodies. Anti-human CCR9 (clone 3C3, mouse IgG2b, produced from hybridoma LS129-3C3-E3-1, ATCC) and anti-human CCR10 (clone 1B5, mouse IgG2a, produced from hybridoma LS312-1B5-1A1, courtesy of Dulce Soler-Ferran, Millenium Pharmaceuticals) were used as described below (cell staining and flow cytometry). Mouse anti-human $\alpha 4 \beta 7$ (clone Act1, mouse IgG1 $\kappa$, from hybridoma Act-1 number 39, a gift from Millenium Pharmaceuticals) was custom-conjugated to PE (Chromoprobe, Inc.). Antibody production from hybridomas was performed as directed by the supplier. Briefly, hybridomas were grown in serum-free medium and antibody purified by ammonium sulfate precipitation from the serum-free culture supernatant. Size-exclusion chromatography was used to determine antibody purity and any protein aggregation or degradation. Recombinant human P-selectin/Ig Fc chimera (R\&D Systems, 137-PS-050) was conjugated to Alexa Fluor 647 using the Zenon Alexa Fluor 647 human Ig bench-top labeling kit (Invitrogen) as directed. In patient cohort 1, CCR9 and CCR10 expression was detected with secondary antibodies goat $\mathrm{F}(\mathrm{ab})_{2}$ anti-mouse Pacific Blue and goat $\mathrm{F}(\mathrm{ab})_{2}$ anti-mouse Qdot 
655, respectively (P31581 and Q-11021MP, Invitrogen). In patient cohort 2, CCR9 and CCR10 were stained separately and each receptor detected with goat $\mathrm{F}(\mathrm{ab})_{2}$ anti-mouse-Pacific Blue. In studies of expression of other receptors by PBMCs from 2 to 5 patients, we used the following purified antibodies: GPR15 (clone 367902) from R\&D Systems; CCR2 (clone K036C2), CXCR5 (clone RF8B2), CD103 (clone BerAct8), $\alpha 2$ (clone P1E6C5) from BioLegend; CCR4 (clone 205410), CCR5 (clone NP-6G4), CXCR4 (clone 12G5) from Fluidigm Sciences. Purified antibodies were conjugated to metal isotopes, and cells were stained and analyzed by mass cytometry as described previously (51).

Primary conjugated antibodies. The following conjugated antibodies were purchased from BD Biosciences: rat anti-human CD49f-PE-Cy5 (clone GoH3), mouse anti-human CD29 (ß1)-PE-Cy5 (clone MAR4), mouse anti-human CXCR3-PerCP-Cy5.5 (clone G025H7), mouse anti-human CD4-V500 (clone RPAT4), mouse anti-human CD19-Alexa Fluor 700 (clone HIB19), and mouse anti-human CD62L-APCCy7 (clone DREG-56). Mouse anti-human CD38-PE-Cy7 (clone HB7) and rat anti-human CLA-biotin (clone HECA-452) from BD Biosciences were provided courtesy of Maria C. Jaimes (BD Biosciences). The following were obtained from Invitrogen: mouse anti-human CD45RA-Qdot 605 (clone MEM-56), polyclonal goat (Fab), anti-human IgA-FITC (clone H14101), and Qdot 565-streptavidin (Q10131MP) to detect CLA-biotin.

Cell staining and flow cytometry. Three million PBMCs per patient sample were stained with the aforementioned antibodies for flow cytometric phenotypic analysis of trafficking receptor profiles. In patient cohort 1, cells were incubated with purified anti-human CCR9, washed in staining buffer, and subsequently stained with secondary goat $\mathrm{F}(\mathrm{ab})_{2}$ anti-mouse Pacific Blue. Cells were washed and then blocked with $7.5 \mu \mathrm{l}$ normal mouse serum (Sigma-Aldrich) for 15 minutes on ice. Primary anti-CCR10 was conjugated to goat $\mathrm{F}(\mathrm{ab})_{2}$ anti-mouse Qdot 655 by combining the primary and secondary and incubating at room temperature for 15 minutes, followed by the addition of mouse serum to block nonspecific binding sites for an additional 15 minutes. A second bench-top conjugation was performed using a Zenon kit as directed to label P-selectin/Ig Fc chimera with Alexa Fluor 647. These bench-top conjugates were added to a cocktail of labeled primary antibodies (IgA-FITC, $\alpha 4 \beta 7-P E$, CD49f-PE-Cy5, CD38-PE-Cy7, CD4-V500, CLA-biotin, CD45RA-Qdot 605, CD19-AF700, and CD62L-APC-Cy7). After staining for 30 minutes at $4^{\circ} \mathrm{C}$, cells were washed twice with staining buffer and Qdot 565-streptavidin was added to detect CLA. In patient cohort 2 , the following changes were made to the staining protocol: CCR9 and CCR10 were stained separately using secondary goat $\mathrm{F}(\mathrm{ab})_{2}$ anti-mouse Pacific Blue as described above for CCR9 and CXCR3-PerCP-Cy5.5 was added to the primary antibody cocktail.

In order to determine background levels and clearly define positive from negative populations, isotype controls were stained as follows: normal mouse serum was added in place of CCR9 and CCR10, P-selectin/Ig binding was disrupted by incubation of the cells with $4 \mu 10.5 \mathrm{M}$ EDTA prior to adding the conjugated antibody cocktail, and both CLA and CD49f were omitted.

All samples were acquired on a BD LSRII flow cytometer using FACSDiva software. Flow cytometric data was analyzed using FlowJo software (Tree Star). Trafficking receptor gates were placed based on the isotype controls and memory $\mathrm{CD} 4^{+} \mathrm{T}$ cells (CD4 ${ }^{+} \mathrm{CD} 45 \mathrm{RA}^{-}$lymphocytes), which serve as a positive control for several markers or a bimodal distribution of positive and negative cell populations.

Cell sorting. PBMCs were isolated from whole blood of TIV or ID-TIV vaccinees as previously described. Cells were incubated with purified anti-human CCR10, washed, and incubated with secondary goat F(ab) anti-mouse Pacific Blue. Cells were washed, blocked with mouse serum, and stained with a cocktail of antibodies: IgA-FITC, CD38-PE-Cy7, CD4-V500, CD19-Alexa Fluor 700, and $\alpha 4 \beta 7-P E$. Within pre-gated $\mathrm{CD}^{-} \mathrm{CD} 38^{\text {hi }}$ single lymphocytes, total $\mathrm{IgA}^{+}$and $\operatorname{IgA}^{-}(\mathrm{IgG}) \mathrm{PBs}$ as well as $\operatorname{IgA}^{+}$and $\operatorname{IgA}^{-}$that were $\alpha 4 \beta 7^{-}$, $\alpha 4 \beta 7^{+}, \alpha 4 \beta 7^{-} \mathrm{CCR} 10^{+}$, and $\alpha 4 \beta 7^{-} \mathrm{CCR} 10^{-}$were sorted (BD FACSAria II or BD FACSAria III) into 96-well nitrocellulose ELISPOT plates. Fifty cells of each phenotype were sorted into triplicate wells. Total IgA/ IgG (ASCs) within each sorted population as well as flu-Ag-specific ASCs were detected and the percentage of flu-Ag-specific ASCs determined as outlined below.

\section{ELISPOT for total and flu-Ag-specific IgA and IgC ASCs}

Total IgA or IgG ASCs were identified by conventional ELISPOT analysis using nitrocellulose 96-well plates (MultiScreen HTS, Millipore) that were coated with goat anti-human-IgM+IgA+IgG (Kirkegaard and Perry) at $4 \mu \mathrm{g} / \mathrm{ml}$ and incubated overnight at $4^{\circ} \mathrm{C}$. To detect flu-Ag-specific ASCs, wells were coated with inactivated influenza vaccine (Fluzone) at $9 \mu \mathrm{g} / \mathrm{ml}$ and incubated overnight at $4^{\circ} \mathrm{C}$. 
The coating solution was discarded and the plates were blocked for at least 2 hours with RPMI 1640 (Mediatech) supplemented with 10\% fetal bovine serum (Hyclone) and 1\% penicillin/streptomycin (Gibco). The media were replaced with fresh blocking media prior to sorting specific trafficking receptor-defined $\operatorname{IgA}^{+}$and $\mathrm{IgA}^{-} \mathrm{PBs}$. After sorting, the plates were incubated overnight at $37^{\circ} \mathrm{C}$ in the presence of $5 \% \mathrm{CO}_{2}$. Cells and media were discarded, plates washed 4 times with $\mathrm{PBS}$ without $\mathrm{Ca}^{++} / \mathrm{Mg}^{++}$, secondary alkaline phosphatase (AP)-conjugated anti-human-IgA or AP-anti-human-IgG (Kirkegaard \& Perry) was added at $0.2 \mu \mathrm{g} / \mathrm{ml}$ and incubated for up to 4 hours at $37^{\circ} \mathrm{C}$. Wells were washed as before and developed using Blue Alkaline Phosphatase Substrate Kit as directed (Vector Labs). Wells were washed with water, allowed to dry, and the number of spots, each representing a functional ASC, was counted under a dissecting microscope. The average number of ASCs of the triplicate wells was calculated for each sorted population and the percent flu-Ag-specific ASCs within each TP-defined subset determined by dividing the number of flu-Ag-specific ASCs by the total number of IgA or IgG ASCs within that population.

\section{Computational analysis}

Data processing. Patient and HD PBs were gated from flow cytometric data as $\mathrm{CD}^{-} \mathrm{CD} 38^{\text {hi }} \operatorname{IgA}^{+}$or $\operatorname{IgA}^{-}$. From each of the 52 samples ( 44 patients and $8 \mathrm{HDs}$ ) included in the study, 500 cells within the $\operatorname{IgA}^{+}$and $\mathrm{IgA}^{-}$gated populations were randomly selected from within FlowJo, totaling close to 52,000 individual cells. The fluorescence intensity of each trafficking receptor on each individual cell was exported as scale values in a tab-delimited text file which was opened within Excel. The 52,000 cells were combined into a single Excel document, and the fluorescent intensity of each trafficking receptor was log transformed in Partek Genomic Suite 6.5 (Partek.com).

$P C A$. PCA was performed on all 7 trafficking receptors and the 3 most influential principal components were selected as axes for the 3D plot (Figure 2A). The dots represent individual cells from the 52 patients and are colored by disease class.

Dimension reduction. tSNE and a variant of $\mathrm{k}$-means clustering were performed using ACCENSE (http://www.cellaccense.com), using a perplexity setting of 500. From the close to 52,000 individual cells exported (see data processing), 99 clusters were defined.

Hierarchical clustering. The number of cells present in each of the 99 clusters, in each individual patient, was determined. Hierarchical clustering was performed on these data using Pearson's dissimilarity without normalization. The data are displayed as a heatmap with the range set from 0 to 100 cells per cluster (Figure 3A).

Similarity matrix. The percentage of cells from each patient that fell within each cluster was calculated and Pearson's (linear) correlation was used to compute the similarity of each individual patient to all other patients as well as the individual patient's similarity to itself (Figure 3B). The resulting similarity is shown as a heatmap whose range is between 0 (no similarity) and 1 (exact similarity).

ANOVA test. $P$ values were calculated using ANOVA, which compares patients within one disease classification to those of the other disease classes as a group. On the tSNE maps and heatmaps, enriched clusters with $P$ less 0.005 and $P$ less than 0.05 are colored as blue and red, respectively. In $2 \mathrm{D}$ plots, the enriched clusters with $P$ less than 0.005 are colored as black (Figures 4 and 5).

Enrichment plot. The percentage of each patient's PBs displaying disease class-associated TPs was calculated and normalized to the average percentage of $\mathrm{PBs}$ from each disease class within the same gated phenotype. By 2 -way ANOVA, the $P$ values between different cell types (IgA ${ }^{+} \mathrm{PBs}, \mathrm{IgA}^{-} \mathrm{PBs}$, and memory IgA cells) were calculated (Figure 6). ${ }^{*} P<0.05 ;{ }^{* *} P<0.005 ;{ }^{* * *} P<0.0005$

\section{Statistics}

Statistical significance of $\operatorname{IgA}^{+}$versus $\operatorname{IgA}^{-}$frequencies within a patient class was determined using paired-ratio, 2-tailed $t$ test. Paired-ratio, 1-tailed $t$ test was used to calculate significance of flu-Ag-specific IgG ASCs among sorted trafficking receptor subsets. tSNE clusters were ordered in heatmaps by unsupervised hierarchical clustering via Pearson's dissimilarity without normalization. Pearson's linear correlation was used to compute the similarity of each individual patient to all other patients as well as the individual patient's similarity to itself. $P$ values of enriched clusters were calculated using ANOVA. Two-way ANOVA was applied when comparing different cell types. $P$ values below 0.05 were considered significant and are indicated in each plot. All dot plots show mean \pm SEM. 


\section{Study approval}

Acute infectious disease patients and healthy subjects were enrolled in the study at the Veterans Affairs Palo Alto Health Care System, with written consent obtained according to Health Insurance Portability and Accountability Act (HIPAA) regulations. Influenza vaccine recipients and celiac disease patients were enrolled after obtaining informed consent as approved by the IRB of Stanford University. Subjects suffering from ulcerative colitis were consented and enrolled through the Kaiser Permanente Foundation Hospital located in San Jose, California.

\section{Author contributions}

YS, NHL, and LS contributed equally to the study and performed all experiments. YS, NHL, and ECB analyzed data and wrote the manuscript. AH and TA provided CeD and UC patient samples. XSH and HBG consulted on the analysis of influenza vaccine samples. ECB oversaw the project and provided overall guidance. All authors provided input on the text.

\section{Acknowledgments}

This study was supported by Gates foundation award OPP1113682 and by NIH grants AI047822, RC1 AI087257, U01 AI089859, and AI090019. H.B. Greenberg was funded by NIH grants AI021362, AI057229, AI115715, and AI090019. A. Habtezion was funded by NIH grant DK101119. The authors thank the blood donors for their participation, Joy Wu and William H. Robinson of the Stanford School of Medicine for helpful discussion and Maria C. Jaimes of BD Biosciences for providing conjugated antibodies used in these studies. We would also like to acknowledge Robert Tibshirani of the Department of Statistics and Health Research and Policy at Stanford University for his statistical consultancy.

Address correspondence to: Eugene C. Butcher, Laboratory of Immunology and Vascular Biology, Veterans Affairs Palo Alto Health Care System, 3801 Miranda Avenue, Building 101, Room B4-145, Palo Alto, California 94304, USA. Phone: 650.852.3369; E-mail: ebutcher@stanford.edu.

1. Jaimes MC, et al. Maturation and trafficking markers on rotavirus-specific B cells during acute infection and convalescence in children. J Virol. 2004;78(20):10967-10976.

2. Wrammert J, et al. Rapid and massive virus-specific plasmablast responses during acute dengue virus infection in humans. J Virol. 2012;86(6):2911-2918.

3. Sinha A, et al. Circulating gut-homing $\left(\alpha 4 \beta 7^{+}\right)$Plasmablast responses against Shigella surface protein antigens among hospitalized patients with diarrhea. Clin Vaccine Immunol. 2016;23(7):610-617.

4. Husband AJ, Gowans JL. The origin and antigen-dependent distribution of IgA-containing cells in the intestine. J Exp Med. 1978;148(5):1146-1160

5. Youngman KR, Franco MA, Kuklin NA, Rott LS, Butcher EC, Greenberg HB. Correlation of tissue distribution, developmental phenotype, and intestinal homing receptor expression of antigen-specific $B$ cells during the murine anti-rotavirus immune response. J Immunol. 2002;168(5):2173-2181.

6. Kunkel EJ, Butcher EC. Plasma-cell homing. Nat Rev Immunol. 2003;3(10):822-829.

7. Cyster JG. Homing of antibody secreting cells. Immunol Rev. 2003;194:48-60.

8. Kuklin NA, et al. Protective intestinal anti-rotavirus B cell immunity is dependent on alpha 4 beta 7 integrin expression but does not require IgA antibody production. J Immunol. 2001;166(3):1894-1902.

9. Wilson E, Butcher EC. CCL28 controls immunoglobulin (Ig)A plasma cell accumulation in the lactating mammary gland and IgA antibody transfer to the neonate. J Exp Med. 2004;200(6):805-809.

10. Lazarus NH, Kunkel EJ, Johnston B, Wilson E, Youngman KR, Butcher EC. A common mucosal chemokine (mucosae-associated epithelial chemokine/CCL28) selectively attracts IgA plasmablasts. J Immunol. 2003;170(7):3799-3805.

11. Masahata K, et al. Generation of colonic IgA-secreting cells in the caecal patch. Nat Commun. 2014;5:3704.

12. Pan J, et al. A novel chemokine ligand for CCR10 and CCR3 expressed by epithelial cells in mucosal tissues. J Immunol. 2000;165(6):2943-2949.

13. Hieshima K, et al. CC chemokine ligands 25 and 28 play essential roles in intestinal extravasation of IgA antibody-secreting cells. J Immunol. 2004;173(6):3668-3675.

14. Bowman EP, et al. The intestinal chemokine thymus-expressed chemokine (CCL25) attracts IgA antibody-secreting cells. $J$ Exp Med. 2002;195(2):269-275.

15. Kantele A, et al. Homing potentials of circulating lymphocytes in humans depend on the site of activation: oral, but not parenteral, typhoid vaccination induces circulating antibody-secreting cells that all bear homing receptors directing them to the gut. J Immunol. 1997;158(2):574-579.

16. Quiding M, Lakew M, Granström G, Nordström I, Holmgren J, Czerkinsky C. Induction of specific antibody responses in the human nasopharyngeal mucosa. Adv Exp Med Biol. 1995;371B:1445-1450.

17. Wrammert J, et al. Rapid cloning of high-affinity human monoclonal antibodies against influenza virus. Nature. 2008;453(7195):667-671. 
18. Abitorabi MA, Mackay CR, Jerome EH, Osorio O, Butcher EC, Erle DJ. Differential expression of homing molecules on recirculating lymphocytes from sheep gut, peripheral, and lung lymph. J Immunol. 1996;156(9):3111-3117.

19. Shekhar K, Brodin P, Davis MM, Chakraborty AK. Automatic classification of cellular expression by nonlinear stochastic embedding (ACCENSE). Proc Natl Acad Sci USA. 2014;111(1):202-207.

20. Amir el-AD, et al. viSNE enables visualization of high dimensional single-cell data and reveals phenotypic heterogeneity of leukemia. Nat Biotechnol. 2013;31(6):545-552.

21. Xu B, et al. Lymphocyte homing to bronchus-associated lymphoid tissue (BALT) is mediated by L-selectin/PNAd, alpha4beta1 integrin/VCAM-1, and LFA-1 adhesion pathways. J Exp Med. 2003;197(10):1255-1267.

22. Curtis JL, Kim S, Scott PJ, Buechner-Maxwell VA. Adhesion receptor phenotypes of murine lung CD4+ $\mathrm{T}$ cells during the pulmonary immune response to sheep erythrocytes. Am J Respir Cell Mol Biol. 1995;12(5):520-530.

23. Rosen SD, Tsay D, Singer MS, Hemmerich S, Abraham WM. Therapeutic targeting of endothelial ligands for L-selectin (PNAd) in a sheep model of asthma. Am J Pathol. 2005;166(3):935-944.

24. McGhee JR, Fujihashi K. Inside the mucosal immune system. PLoS Biol. 2012;10(9):e1001397.

25. Bargatze RF, Jutila MA, Butcher EC. Distinct roles of L-selectin and integrins alpha 4 beta 7 and LFA-1 in lymphocyte homing to Peyer's patch-HEV in situ: the multistep model confirmed and refined. Immunity. 1995;3(1):99-108.

26. Berlin C, et al. Alpha 4 integrins mediate lymphocyte attachment and rolling under physiologic flow. Cell. 1995;80(3):413-422.

27. Kunkel EJ, et al. Lymphocyte CC chemokine receptor 9 and epithelial thymus-expressed chemokine (TECK) expression distinguish the small intestinal immune compartment: Epithelial expression of tissue-specific chemokines as an organizing principle in regional immunity. J Exp Med. 2000;192(5):761-768.

28. Gironella M, et al. The role of P-selectin in experimental colitis as determined by antibody immunoblockade and genetically deficient mice. J Leukoc Biol. 2002;72(1):56-64.

29. Sperandio M, Gleissner CA, Ley K. Glycosylation in immune cell trafficking. Immunol Rev. 2009;230(1):97-113.

30. Ley K, Laudanna C, Cybulsky MI, Nourshargh S. Getting to the site of inflammation: the leukocyte adhesion cascade updated. Nat Rev Immunol. 2007;7(9):678-689.

31. Palkola NV, Blomgren K, Pakkanen SH, Puohiniemi R, Kantele JM, Kantele A. Immune defense in upper airways: a single-cell study of pathogen-specific plasmablasts and their migratory potentials in acute sinusitis and tonsillitis. PLoS One. 2016;11(4):e0154594.

32. Bochner BS. Road signs guiding leukocytes along the inflammation superhighway. J Allergy Clin Immunol. 2000;106(5):817-828.

33. Henderson WR, et al. Blockade of CD49d (alpha4 integrin) on intrapulmonary but not circulating leukocytes inhibits airway inflammation and hyperresponsiveness in a mouse model of asthma. J Clin Invest. 1997;100(12):3083-3092.

34. Berg EL, et al. The cutaneous lymphocyte antigen is a skin lymphocyte homing receptor for the vascular lectin endothelial cellleukocyte adhesion molecule 1. J Exp Med. 1991;174(6):1461-1466.

35. Moughal NA, Adonogianaki E, Thornhill MH, Kinane DF. Endothelial cell leukocyte adhesion molecule-1 (ELAM-1) and intercellular adhesion molecule-1 (ICAM-1) expression in gingival tissue during health and experimentally-induced gingivitis. J Periodont Res. 1992;27(6):623-630.

36. Tonetti MS, Gerber L, Lang NP. Vascular adhesion molecules and initial development of inflammation in clinically healthy human keratinized mucosa around teeth and osseointegrated implants. J Periodont Res. 1994;29(6):386-392.

37. Picker LJ, Kishimoto TK, Smith CW, Warnock RA, Butcher EC. ELAM-1 is an adhesion molecule for skin-homing T cells. Nature. 1991;349(6312):796-799.

38. Czerkinsky C, Svennerholm AM, Quiding M, Jonsson R, Holmgren J. Antibody-producing cells in peripheral blood and salivary glands after oral cholera vaccination of humans. Infect Immun. 1991;59(3):996-1001.

39. Tarlton NJ, et al. Plasmablast frequency and trafficking receptor expression are altered in pediatric ulcerative colitis. Inflamm Bowel Dis. 2012;18(12):2381-2391.

40. Rott LS, Briskin MJ, Butcher EC. Expression of alpha4beta7 and E-selectin ligand by circulating memory B cells: implications for targeted trafficking to mucosal and systemic sites. J Leukoc Biol. 2000;68(6):807-814.

41. Bowman EP, et al. Developmental switches in chemokine response profiles during B cell differentiation and maturation. $J$ Exp Med. 2000;191(8):1303-1318.

42. Lee AY, Eri R, Lyons AB, Grimm MC, Korner H. CC Chemokine ligand 20 and its cognate receptor CCR6 in mucosal t cell immunology and inflammatory bowel disease: Odd couple or axis of evil? Front Immunol. 2013;4:194.

43. Johansson C, Ahlstedt I, Furubacka S, Johnsson E, Agace WW, Quiding-Järbrink M. Differential expression of chemokine receptors on human IgA ${ }^{+}$and $\mathrm{IgG}^{+}$B cells. Clin Exp Immunol. 2005;141(2):279-287.

44. Nishiuchi R, et al. Characterization of the ligand-binding specificities of integrin alpha3beta 1 and alpha6beta 1 using a panel of purified laminin isoforms containing distinct alpha chains. J Biochem. 2003;134(4):497-504.

45. Song J, et al. Extracellular matrix of secondary lymphoid organs impacts on B-cell fate and survival. Proc Natl Acad Sci USA. 2013;110(31):E2915-E2924

46. Schaff $\mathrm{M}$, et al. Integrin $\alpha 6 \beta 1$ is the main receptor for vascular laminins and plays a role in platelet adhesion, activation, and arterial thrombosis. Circulation. 2013;128(5):541-552.

47. Lahl K, Sweere J, Pan J, Butcher E. Orphan chemoattractant receptor GPR15 mediates dendritic epidermal T-cell recruitment to the skin. Eur J Immunol. 2014;44(9):2577-2581.

48. Nguyen LP, et al. Role and species-specific expression of colon T cell homing receptor GPR15 in colitis. Nat Immunol. $2015 ; 16(2): 207-213$.

49. Kim SV, et al. GPR15-mediated homing controls immune homeostasis in the large intestine mucosa. Science. 2013;340(6139):1456-1459.

50. Turner D, et al. Development, validation, and evaluation of a pediatric ulcerative colitis activity index: a prospective multicenter study. Gastroenterology. 2007;133(2):423-432.

51. Bendall SC, et al. Single-cell mass cytometry of differential immune and drug responses across a human hematopoietic continuum. Science. 2011;332(6030):687-696. 